\title{
High- and low-impact citation measures: Empirical applications
}

\author{
Pedro Albarrán, Ignacio Ortuño, Javier Ruiz-Castillo* \\ Departamento de Economía, Universidad Carlos III, Madrid 128, Getafe 28903, Spain
}

\section{A R T I C L E I N F O}

\section{Article history:}

Received 26 March 2010

Received in revised form 12 August 2010

Accepted 4 October 2010

\section{Key words:}

Research evaluation

Citation distribution

Scientific ranking

Impact indicators

\begin{abstract}
A B S T R A C T
This paper contains the first empirical applications of a novel methodology for comparing the citation distributions of research units working in the same homogeneous field. The paper considers a situation in which the world citation distribution in 22 scientific fields is partitioned into three geographical areas: the U.S., the European Union (EU), and the rest of the world $(\mathrm{RW})$. Given a critical citation level $(\mathrm{CCL})$, we suggest using two real valued indicators to describe the shape of each area's distribution: a high- and a low-impact measure defined over the set of articles with citations below or above the CCL. It is found that, when the CCL is fixed at the 80th percentile of the world citation distribution, the U.S. performs dramatically better than the EU and the RW according to both indicators in all scientific fields. This superiority generally increases as we move from the incidence to the intensity and the citation inequality aspects of the phenomena in question. Surprisingly, changes observed when the CCL is increased from the 80th to the 95th percentile are of a relatively small order of magnitude. Finally, it is found that international co-authorship increases the high-impact and reduces the low-impact level in the three geographical areas. This is especially the case for the EU and the RW when they cooperate with the U.S.
\end{abstract}

\section{Introduction}

In Albarrán, Ortuño, and Ruiz-Castillo (2011), we presented a novel methodology for the evaluation of the scientific performance of research units of a certain size working in the same homogeneous field, namely, a scientific field where the number of citations received by any two papers is comparable independently of the journal in which they have been published. It is well known that citation distributions are highly skewed. Correspondingly, the upper and the lower part of any citation distribution are typically very different. This is the reason why, given a critical citation level (CCL hereafter), we suggest using two indicators to describe this key feature of a citation distribution: a high-and a low-impact measure defined over the sets of articles with citations above and below the CCL.

This paper contains the first empirical applications of such an approach to a situation in which the world citation distribution in a given field is partitioned into three geographical areas: articles with at least one author working in a research institution (i) in the U.S.; (ii) in the EU, namely, the 15 countries forming the European Union before the 2004 accession, or (iii) in any other country of the rest of the world (RW hereafter). For that purpose, we use a large sample acquired from Thomson Scientific (TS) consisting of 3.6 million articles published in 1998-2002, as well as the more than 28 million citations they receive when a five-year citation window is used. We focus on the case in which homogeneous fields are identified with the 20 natural sciences and the 2 social sciences distinguished by TS. The CCL in each field is set equal to the number of citations received by papers in the 80th percentile of the world citation distribution of the field in question.

\footnotetext{
* Corresponding author.Tel.: +349162495 88; fax: +34916249329.

E-mail address: jrc@eco.uc3m.es (J. Ruiz-Castillo).
} 
Borrowing results from the economic literature, Albarrán, Ortuño, and Ruiz-Castillo (2010c) show that the ranking induced by a family of low-impact measures that satisfy a number of basic and other admissible properties essentially coincide with that obtained from a family of indices originally suggested by Foster, Greeer, and Thorbecke (1984) for the measurement of economic poverty. Those same properties lead to the selection of an equally convenient class of decomposable high-impact measures that is the counterpart of the family just mentioned. Moreover, the two families in question that will be referred to as the FGT high- and low-impact families - satisfy a number of other properties that might be useful in practice.

In this paper we use three members of the FGT families that capture different dimensions of the phenomena to be measured. To appreciate this point, let us focus for a moment on the measurement of high-impact in the U.S. citation distribution in a certain scientific field. The first member of the FGT family is equal to the percentage of high-impact papers in the field that have been written in the U.S., capturing what we call the incidence of the high-impact phenomenon. In addition, the second member of the family incorporates a measure of the aggregate gap between the actual number of citations received by each high-impact paper in the U.S. and the CCL, that is, a measure of the intensity of the phenomenon in question. Finally, together with the incidence and the intensity, the third member of the family includes a measure of the citation inequality among U.S. high-impact papers ${ }^{1}$. The empirical questions studied in this first application of our methodology are the following four:

(i) How does the situation of each geographical area in each field vary when, given a CCL, the incidence, the intensity and the inequality aspects of the high- and low-impact characteristics of their citation distributions are successively taken into account?

(ii) What is the relationship, if any, between high- and low-impact levels and publishing shares across areas in each field, and between high- and low-impact levels and publishing efforts across fields in each area?

(iii) How does the high- and low-impact relative situation of each area in each field vary when the CCL is increased?

(iv) Given a CCL, is it the case that different types of international co-authorship always improve the scientific performance of any geographical area by raising the high-impact measure and/or lowering the low-impact indicator? Which geographical area is more dependent on the good performance of internationally co-authored papers?

The rest of this paper is organized into four sections and an Appendix. Section 2 introduces the FGT families of high- and low-impact indicators that will be used in the empirical part. Section 3 presents the data, while some basic computations are relegated to Appendix A. Section 4 contains the empirical findings about the scientific performance of the U.S., the EU, and the RW in 22 homogeneous fields, including the effect of international co-authorship in each geographical area. Finally, Section 5 discusses the results and offers some conclusions.

\section{Notation and definitions}

\subsection{Notation}

A discrete citation distribution of papers published in a given year is a non-negative vector $\boldsymbol{x}=\left(x_{1}, \ldots, x_{i,}, \ldots, x_{n}\right)$, where $x_{i} \geq 0$ is the number of citations received by the $i$ th article over a certain number of years since its publication date - a period known as the citation window. Given a distribution $\boldsymbol{x}$ and a positive CCL, $z>0$, classify as low- or high-impact articles all papers with citation $x_{i} \leq z$, or $x_{i}>z$. Denote by $n(\boldsymbol{x})$ the total number of articles in the distribution, and by $l(\boldsymbol{x} ; z)$ and $h(\boldsymbol{x}$; $z)=n(\boldsymbol{x})-l(\boldsymbol{x} ; z)$ the number of low- and high-impact articles. A low-impact index is a real valued function $L$ whose typical value $L(\boldsymbol{x} ; z)$ indicates the low-impact level associated with the distribution $\boldsymbol{x}$ and the CCL $z$, while a high-impact index is a real valued function $H$ whose typical value $H(\boldsymbol{x} ; z)$ indicates the high-impact level associated with the distribution $\boldsymbol{x}$ and the CCL $z$.

Given a citation distribution $\boldsymbol{x}$ and a CCL $z$, define the normalized low-impact gap for any article with $x_{i}$ citations by

$$
\Gamma_{i}=\max \left\{\frac{\left(z-x_{i}\right)}{z}, 0\right\}
$$

Thus, $\Gamma_{i} \geq 0$ for low-impact articles, while $\Gamma_{i}=0$ for high-impact articles. Similarly, define the normalized high-impact gap by

$$
\Gamma_{i}=\max \left\{\frac{\left(x_{i}-z\right)}{z}, 0\right\}
$$

Thus, $\Gamma_{i}^{*}>0$ for high-impact articles, while $\Gamma_{i}^{*}=0$ for low-impact articles.

\footnotetext{
${ }^{1}$ Egghe, Rousseau, and Rousseau (2007) develop a methodology -TOP-Curves- to provide an (incomplete) ranking of citation distributions that takes into account the incidence, the intensity, and the citation inequality dimensions of their high-impact articles. Rousseau, Verbeke, and Rousseau (2009) contains the first empirical application of this methodology in scientometrics for the evaluation of environmental and resource economics journals.
} 


\subsubsection{The FGT family of low- and high-impact indicators}

The FGT family of low-impact indicators, originally introduced in Foster, Greeer, and Thorbecke (1984) for the measurement of economic poverty, is a function of normalized low-impact gaps defined by

$$
L_{\beta}(\boldsymbol{x} ; z)=\left[\frac{1}{n(\boldsymbol{x})}\right] \Sigma_{i=1}^{l(\boldsymbol{x} ; z)}\left(\Gamma_{i}\right)^{\beta}, 0 \leq \beta .
$$

The class of FGT high-impact indicators is a function of normalized high-impact gaps defined by

$$
H_{\beta}(\boldsymbol{x} ; z)=\left[\frac{1}{n(\boldsymbol{x})}\right] \Sigma_{i=l(\boldsymbol{x} ; z)}^{n(\boldsymbol{x})}\left(\Gamma_{i}^{*}\right)^{\beta}, 0 \leq \beta
$$

It will be sufficient to understand the differences involved in the use of the members of these two classes for parameter values $\beta=0,1$, and 2 . Firstly, note that the high- and low-impact indices obtained when $\beta=0$ coincide with the proportion of high- or low-impact papers ${ }^{2}$ :

$$
H_{0}(\boldsymbol{x} ; z)=\frac{h(\boldsymbol{x} ; z)}{n(\boldsymbol{x})}
$$

and

$$
L_{0}(\boldsymbol{x} ; z)=\frac{l(\boldsymbol{x} ; z)}{n(\boldsymbol{x})}
$$

Of course, $H_{0}(\boldsymbol{x} ; z)+L_{0}(\boldsymbol{x} ; z)=1$, so that if $H_{0}(\boldsymbol{x} ; z)$ changes, then $L_{0}(\boldsymbol{x} ; z)$ must change in the opposite direction.

Secondly, consider the high-impact index corresponding to the parameter value $\beta=1$, or the per-article high-impact gap ratio:

$$
H_{1}(\boldsymbol{x} ; z)=\left[\frac{1}{n(\boldsymbol{x})}\right] \sum_{i=l(\boldsymbol{x} ; z)+1}^{n(\boldsymbol{x})} \Gamma_{i}^{*}
$$

This convenient high-impact indicator represents the surplus of citations actually received by high-impact articles above the CCL. Similarly, the member of the FGT family of low-impact indicators for $\beta=1$, or the per-article low-impact gap ratio, is equal to

$$
L_{1}(\boldsymbol{x} ; z)=\left[\frac{1}{n(\boldsymbol{x})}\right]\left[\sum_{i=1}^{l(\boldsymbol{x} ; z)} \Gamma_{i}\right]
$$

This low-impact indicator represents the minimum number of citations required to bring all low-impact articles to the CCL. Denote by $\mu_{H}(\boldsymbol{x})$ and $\mu_{L}(\boldsymbol{x})$ the MCR of high- and low-impact articles. It can be shown that $H_{1}(\boldsymbol{x} ; z)=H_{0}(\boldsymbol{x} ; z) H_{I}(\boldsymbol{x} ; z)$ and $L_{1}(\boldsymbol{x}$; $z)=L_{0}(\boldsymbol{x} ; z) L_{I}(\boldsymbol{x} ; z)$, where

$$
H_{I}(\boldsymbol{x} ; z)=\left[\frac{1}{h(\boldsymbol{x} ; z)}\right] \sum_{i=l(\boldsymbol{x} ; z)+1}^{n(\boldsymbol{x})} \Gamma_{i}^{*}=\frac{\left[\mu_{H}(\boldsymbol{x})-z\right]}{z}
$$

and

$$
L_{I}(\boldsymbol{x} ; z)=\left[\frac{1}{l(\boldsymbol{x} ; z)}\right] \sum_{i=1}^{l(\boldsymbol{x} ; z)} \Gamma_{i}=\frac{\left[z-\mu_{L}(\boldsymbol{x})\right]}{z}
$$

The indices $H_{I}$ and $L_{I}$ are said to be monotonic in the sense that one more citation among high-or low-impact articles increases $H_{I}$ or decreases $L_{I}$. Therefore, while $H_{0}$ and $L_{0}$ only capture what we have called the incidence of the high-and low-impact aspects of any citation distribution, $H_{1}$ and $L_{1}$ capture both the incidence and the intensity of these phenomena.Thirdly, the high- and low-impact members of the FGT families obtained when $\beta=2$ can be expressed as

$$
\begin{aligned}
& H_{2}(\boldsymbol{x} ; z)=H_{0}(\boldsymbol{x} ; z)\left\{\left[\left(H_{1}(\boldsymbol{x} ; z)\right]^{2}+\left[1-H_{1}(\boldsymbol{x} ; z)\right]^{2}\left(C_{H}\right)^{2}\right]\right\}, \\
& L_{2}(\boldsymbol{x} ; z)=L_{0}(\boldsymbol{x} ; z)\left\{\left[\left(L_{0}(\boldsymbol{x} ; z)\right]^{2}+\left[1-L_{1}(\boldsymbol{x} ; z)\right]^{2}\left(C_{L}\right)^{2}\right]\right\},
\end{aligned}
$$

where $\left(C_{H}\right)^{2}$ and $\left(C_{L}\right)^{2}$ are the squared coefficient of variation (that is, the ratio of the standard deviation over the mean) among the low- and high-impact articles, respectively. Therefore, $H_{2}$ and $L_{2}$ simultaneously cover the incidence, the intensity, and the citation inequality aspects of the high- and low-impact phenomenon they measure (for other properties of the FGT families of indicators, see Section 3 and Table 2 in Albarrán et al., 2010c).

\footnotetext{
2 It should be observed that many common indices widely used in the income poverty literature, which in our context can be taken as low-impact indicators, are also functions of the normalized low-impact gaps (see footnote 19 in Albarrán et al., 2010c). Furthermore, it is not difficult to convert low-impact indices into high-impact ones as we have done for the original FGT family.
} 


\section{A description of the data}

\subsection{The sample}

TS indexed journal articles include research articles, reviews, proceedings papers and research notes. In this paper, only research articles, or simply articles, are studied. The key assumption that permits the linkage between theoretical concepts and the data is the identification of the 20 natural sciences and the two social sciences distinguished by TS with the homogeneous fields defined in Section 1. We are interested in solidly establishing the relative situation of three large geographical areas - the U.S., the EU, and the RW - in all fields. Since many of them are rather small (nine of the fields represent less than $2 \%$ of the total, and another five between $2 \%$ and $3 \%$ ), the computation of statistically reliable indicators of scientific performance in the smaller ones requires a sizable sample. Therefore, after the elimination of observations with missing values for some variables, the empirical exercise conducted in this paper refers to 3,654,675 articles published in 1998-2002. A five-year citation window has been selected for all fields, so that articles published in 1998 receive citations during the 1998-2002 period, articles published in 1999 receive citations in the 1999-2003 period, etc. The total number of citations amount to $28,296,113$.

\subsection{The assignment of articles to geographical areas}

In any field, an article might be written by one or more scientists working in only one of the three geographical areas, or it might be co-authored by scientists working in two or three of them. The partitions of each field's articles into the seven possible sub-groups, as well as the percentage distribution of the total number of articles by field, are presented in Table 1. The 20 fields in the natural sciences are organized in three large aggregates: Life Sciences, Physical Sciences, and Other Natural Sciences. The last two represent, approximately, $28.5 \%$ and $25.5 \%$ of the total, while the Life Sciences represent about 41\%. The remaining 5\% corresponds to the two Social Sciences (column 9 in Table 1).

Not surprisingly the degree of international co-authorship (columns 4-7 in Table 1) is largest in Space Science where it represents 33.4\% of the total. In six fields (Mathematics, Microbiology, Molecular Biology and Genetics, Physics, and Geosciences) this percentage is approximately between $15 \%$ and $20 \%$, while in eight fields (Social Sciences, Psychiatry and Psychology, Agricultural Sciences, Multidisciplinary, Pharmacology and Toxicology, Materials Science, Chemistry, and Engineering) international co-authorship is relatively less important representing only between $5 \%$ and $11 \%$ of the total. For all sciences as a whole, the percentage of internationally co-authored articles is $12.8 \%$; the most important type is the co-authorship between the EU and the RW with a 5.2\% percentage. As will be seen in Section 4.4, these relatively small percentages of internationally co-authored articles play a crucial role in most fields.

Articles are assigned to geographical areas according to the institutional affiliation of their authors as recorded in the TS database on the basis of what had been indicated in the by-line of the publications. The assignment of internationally co-authored papers among areas is problematic (For a discussion, see inter alia Anderson et al., 1988). From a U.S. geopolitical point of view, for example, we want to give equal weight to an article written in a U.S. research center as we give to another co-authored by researchers from a U.S. and a European university. Thus, as in the classical studies by May (1997) and King (2004), for most purposes in this paper in every internationally co-authored article a whole count is credited to each contributing area. Therefore, articles co-authored by one or more scientists affiliated to institutions in two areas are counted twice, while articles co-authored by persons in the three areas are counted three times. Only domestic articles, or articles exclusively authored by one or more scientists affiliated to research centers either in the U.S., the EU, or the RW alone, are counted once. The total number of articles in such extended count is 4,150,577, or 13,6\% more than the standard count where all articles are counted once. Similarly, the total number of citations in the extended sample is $20.2 \%$ greater than the one in the standard dataset.

Table 2 informs about the percentage distribution of the extended number of articles by field and by geographical area. It is observed that the world distribution of extended articles (column 4) is rather close to the original one (column 9 in Table 1 ). On the other hand, the domestically produced articles in the U.S., the EU, and the RW represent $25,4 \%, 27,8 \%$, and $34.1 \%$ of the total in the original distribution (last row for columns 1-3 in Table 1), while in the extended count, these percentages become $29 \%, 32.3 \%$, and $38.7 \%$ (last row for columns $5-7$ in Table 2 ).

\subsection{The choice of the CCL}

In economics, there is a general agreement that the measurement of economic poverty involves an irreducible, absolute core that should be addressed by fixing an absolute poverty line common to all countries in the world. ${ }^{3}$ However, after World War II it was observed that, at any reasonable absolute poverty line, there would be no absolute poverty in the developed part of the world. Therefore, a notion of relative poverty was introduced where the poverty line is fixed at a certain percentage typically $50 \%$ or $60 \%$ - of mean or median income.

\footnotetext{
${ }^{3}$ At present, the World Bank establishes that absolute poverty line at two dollars per day of equivalent purchasing power in any country of the world.
} 
Table 1

Number of articles and publication shares by authorship type in every scientific field, and percentage distribution of all articles published in 1998-2002 by scientific field.

\begin{tabular}{|c|c|c|c|c|c|c|c|c|c|}
\hline & $\begin{array}{l}\text { U.S. } \\
(1)\end{array}$ & $\begin{array}{l}\mathrm{EU} \\
(2)\end{array}$ & $\begin{array}{l}\text { RW } \\
(3)\end{array}$ & $\begin{array}{l}\text { US + EU } \\
(4)\end{array}$ & $\begin{array}{l}\text { US + RW } \\
\text { (5) }\end{array}$ & $\begin{array}{l}E U+R W+R W \\
(6)\end{array}$ & $\begin{array}{l}\text { Total } \\
(7)\end{array}$ & $\begin{array}{l}\mathrm{US}+\mathrm{EU} \\
(8)\end{array}$ & $\begin{array}{l}\text { Percentage distribution } \\
\text { (9) }\end{array}$ \\
\hline \multirow[t]{2}{*}{ Life sciences } & 449,031 & 466,745 & 399,054 & 48,698 & 56,381 & 56,687 & 11,260 & $1,487,856$ & 40.7 \\
\hline & 30.2 & 31.4 & 26.8 & 3.3 & 3.8 & 3.8 & 0.8 & 100.0 & \\
\hline \multirow[t]{2}{*}{ (1) Clinical medicine } & 229,151 & 267,856 & 208,310 & 20,442 & 25,050 & 23,655 & 5,133 & 779,597 & 21.3 \\
\hline & 29.4 & 34.4 & 26.7 & 2.6 & 3.2 & 3.0 & 0.7 & 100.0 & \\
\hline \multirow[t]{2}{*}{ (2) Biology and biochemistry } & 61,530 & 61,803 & 70,828 & 8,716 & 10,552 & 11,660 & 1,762 & 226,851 & 6.2 \\
\hline & 27.1 & 27.2 & 31.2 & 3.8 & 4.7 & 5.1 & 0.8 & 100.0 & \\
\hline \multirow[t]{2}{*}{ (3) Neuroscience and behav. science } & 36,690 & 34,576 & 28,270 & 4,907 & 4,878 & 4,939 & 939 & 115,199 & 3.2 \\
\hline & 31.8 & 30.0 & 24.5 & 4.3 & 4.2 & 4.3 & 0.8 & 100.0 & \\
\hline \multirow[t]{2}{*}{ (4) Molecular biology and genetics } & 32,590 & 26,951 & 23,442 & 5,934 & 5,464 & 5,278 & 1,553 & 101,212 & 2.8 \\
\hline & 32.2 & 26.6 & 23.2 & 5.9 & 5.4 & 5.2 & 1.5 & 100.0 & \\
\hline \multirow[t]{2}{*}{ (5) Psychiatry and psychology } & 43,872 & 23,302 & 15,960 & 2,227 & 3,049 & 1,867 & 342 & 90,619 & 2.5 \\
\hline & 48.4 & 25.7 & 17.6 & 2.5 & 3.4 & 2.1 & 0.4 & 100.0 & \\
\hline \multirow[t]{2}{*}{ (6) Pharmacology and toxicology } & 14,573 & 18,048 & 23,854 & 1,627 & 2,000 & 2,722 & 279 & 63,103 & 1.7 \\
\hline & 23.1 & 28.6 & 37.8 & 2.6 & 3.2 & 4.3 & 0.4 & 100.0 & \\
\hline \multirow[t]{2}{*}{ (7) Microbiology } & 14,764 & 19,083 & 17,544 & 2,273 & 2,397 & 3,656 & 533 & 60,250 & 1.6 \\
\hline & 24.5 & 31.7 & 29.1 & 3.8 & 4.0 & 6.1 & 0.9 & 100.0 & \\
\hline \multirow{2}{*}{ (8) Immunology } & 15,861 & 15,126 & 10,846 & 2,572 & 2,991 & 2,910 & 719 & 51,025 & 1.4 \\
\hline & 31.1 & 29.6 & 21.3 & 5.0 & 5.9 & 5.7 & 1.4 & 100.0 & \\
\hline \multirow[t]{2}{*}{ Physical sciences } & 176,640 & 265,806 & 433,427 & 32,867 & 40,928 & 78,376 & 12,053 & $1,040,097$ & 28.5 \\
\hline & 17.0 & 25.6 & 41.7 & 3.2 & 3.9 & 7.5 & 1.2 & 100.0 & \\
\hline \multirow[t]{2}{*}{ (9) Chemistry } & 71,825 & 118,596 & 211,010 & 8,400 & 11,568 & 27,008 & 1,838 & 450,245 & 12.3 \\
\hline & 16.0 & 26.3 & 46.9 & 1.9 & 2.6 & 6.0 & 0.4 & 100.0 & \\
\hline \multirow[t]{2}{*}{ (10) Physics } & 55,877 & 87,048 & 157,008 & 13,075 & 16,921 & 36,795 & 6,524 & 373,248 & 10.2 \\
\hline & 15.0 & 23.3 & 42.1 & 3.5 & 4.5 & 9.9 & 1.7 & 100.0 & \\
\hline \multirow[t]{2}{*}{ (11) Computer science } & 19,199 & 21,989 & 21,823 & 2,291 & 3,352 & 2,775 & 405 & 71,834 & 2.0 \\
\hline & 26.7 & 30.6 & 30.4 & 3.2 & 4.7 & 3.9 & 0.6 & 100.0 & \\
\hline \multirow[t]{2}{*}{ (12) Mathematics } & 19,209 & 26,612 & 32,866 & 3,784 & 5,420 & 6,966 & 697 & 95,554 & 2.6 \\
\hline & 20.1 & 27.9 & 34.4 & 4.0 & 5.7 & 7.3 & 0.7 & 100.0 & \\
\hline \multirow[t]{2}{*}{ (13) Space SCIENCE } & 10,530 & 11,561 & 10,720 & 5,317 & 3,667 & 4,832 & 2,589 & 49,216 & 1.3 \\
\hline & 21.4 & 23.5 & 21.8 & 10.8 & 7.5 & 9.8 & 5.3 & 100.0 & \\
\hline \multirow[t]{2}{*}{ Other natural scis } & 205,404 & 238,439 & 376,848 & 19,586 & 37,633 & 52,777 & 5,364 & 936,051 & 25.6 \\
\hline & 21.9 & 25.5 & 40.3 & 2.1 & 4.0 & 5.6 & 0.6 & 100.0 & \\
\hline \multirow[t]{2}{*}{ (14) Engineering } & 67,108 & 71,786 & 117,582 & 5,914 & 11,224 & 12,817 & 1,319 & 287,750 & 7.9 \\
\hline & 23.3 & 24.9 & 40.9 & 2.1 & 3.9 & 4.5 & 0.5 & 100.0 & \\
\hline (15) Plant and animal science & 50,103 & 55,569 & 82,138 & 4,030 & 9,044 & 13,019 & 1,153 & 215,056 & 5.9 \\
\hline & 23.3 & 25.8 & 38.2 & 1.9 & 4.2 & 6.1 & 0.5 & 100.0 & \\
\hline (16) Materials science & 23,549 & 39,836 & 81,287 & 2,466 & 4,660 & 9,809 & 536 & 162,143 & 4.4 \\
\hline & 14.5 & 24.6 & 50.1 & 1.5 & 2.9 & 6.0 & 0.3 & 100.0 & \\
\hline (17) Geoscience & 21,798 & 23,641 & 31,616 & 4,251 & 5,808 & 8,165 & 1,493 & 96,772 & 2.6 \\
\hline & 22.5 & 24.4 & 32.7 & 4.4 & 6.0 & 8.4 & 1.5 & 100.0 & \\
\hline (18) Environment and ecology & 25,592 & 24,092 & 27,104 & 1,746 & 4,187 & 5,253 & 593 & 88,567 & 2.4 \\
\hline & 28.9 & 27.2 & 30.6 & 2.0 & 4.7 & 5.9 & 0.7 & 100.0 & \\
\hline (19) Agricultural sciences & 13,452 & 20,784 & 26,726 & 823 & 2,108 & 3,043 & 174 & 67,110 & 1.8 \\
\hline & 20.0 & 31.0 & 39.8 & 1.2 & 3.1 & 4.5 & 0.3 & 100.0 & \\
\hline (20) Multidisciplinary & 3,802 & 2,731 & 10,395 & 356 & 602 & 671 & 96 & 18,653 & 0.5 \\
\hline & 20.4 & 14.6 & 55.7 & 1.9 & 3.2 & 3.6 & 0.5 & 100.0 & \\
\hline Social sciences & 96,280 & 44,727 & 35,558 & 3,649 & 6,131 & 3,817 & 509 & 190,671 & 5.2 \\
\hline & 50.5 & 23.5 & 18.6 & 1.9 & 3.2 & 2.0 & 0.3 & 100.0 & \\
\hline (21) Social sciences, general & 73,746 & 31,042 & 26,713 & 1,544 & 3,372 & 2,305 & 254 & 138,976 & 3.8 \\
\hline & 53.1 & 22.3 & 19.2 & 1.1 & 2.4 & 1.7 & 0.2 & 100.0 & \\
\hline (22) Economics and business & 22,534 & 13,685 & 8,845 & 2,105 & 2,759 & 1,512 & 255 & 51,695 & 1.4 \\
\hline & 43.6 & 26.5 & 17.1 & 4.1 & 5.3 & 2.9 & 0.5 & 100.0 & \\
\hline All sciences & 927,355 & $1,015,717$ & $1,244,887$ & 104,800 & 141,073 & 191,657 & 29,186 & $3,654,675$ & 100.0 \\
\hline & 25.4 & 27.8 & 34.1 & 2.9 & 3.9 & 5.2 & 0.8 & 100.0 & \\
\hline
\end{tabular}

In citation space, there are also two alternatives in every homogeneous field. Firstly, a relative approach in which a CCL for each geographical area is fixed, for instance, as a multiple of the mean or the median, or at a given percentile of the area's citation distribution. Secondly, an absolute approach in which a CCL for the entire field is fixed as a function of some characteristic of the world citation distribution. In our experience, it is generally agreed that what happens at the world level in any scientific field constitutes a natural reference for the evaluation of the performance of any type of research unit in that field. Therefore, we suggest fixing the CCL at some percentile of the original world distribution in every science. Taking into account the skewness of citation distributions, this paper studies the cases where the CCL is fixed at the 80th or the 95th percentiles. Table 3 informs about the absolute number of citations, the multiple of the mean that this number represents, and the percentage of the total number of citations received by the high-impact articles in each case. 
Table 2

The classification of extended articles by scientific field and geographical area, 1998-2002.

\begin{tabular}{|c|c|c|c|c|c|c|c|c|}
\hline & \multicolumn{4}{|c|}{ Percentage distribution by scientific field } & \multicolumn{4}{|c|}{ Percentage distribution by geographical area } \\
\hline & $\begin{array}{l}\text { U.S. } \\
\text { (1) }\end{array}$ & $\begin{array}{l}\text { EU } \\
(2)\end{array}$ & $\begin{array}{l}\text { RW } \\
\text { (3) }\end{array}$ & $\begin{array}{l}\text { Total } \\
\text { (4) }\end{array}$ & $\begin{array}{l}\text { U.S. } \\
(5)\end{array}$ & $\begin{array}{l}\text { EU } \\
(6)\end{array}$ & $\begin{array}{l}\text { RW } \\
\text { (7) }\end{array}$ & $\begin{array}{l}\text { Total } \\
\text { (8) }\end{array}$ \\
\hline Life sciences & 47.0 & 43.5 & 32.6 & 40.3 & 33.8 & 34.9 & 31.3 & 100.0 \\
\hline (1) Clinical medicine & 23.3 & 23.6 & 16.3 & 20.7 & 32.6 & 36.9 & 30.5 & 100.0 \\
\hline (2) Biology and biochemistry & 6.9 & 6.3 & 5.9 & 6.3 & 31.6 & 32.1 & 36.3 & 100.0 \\
\hline (3) Neuroscience and behav. science & 3.9 & 3.4 & 2.4 & 3.2 & 36.0 & 34.4 & 29.6 & 100.0 \\
\hline (4) Molecular biology and genetics & 3.8 & 3.0 & 2.2 & 2.9 & 37.6 & 32.8 & 29.5 & 100.0 \\
\hline (5) Psychiatry and psychology & 4.1 & 2.1 & 1.3 & 2.4 & 50.3 & 28.2 & 21.6 & 100.0 \\
\hline (6) Pharmacology and toxicology & 1.5 & 1.7 & 1.8 & 1.7 & 26.4 & 32.4 & 41.2 & 100.0 \\
\hline (7) Microbiology & 1.7 & 1.9 & 1.5 & 1.7 & 28.7 & 36.7 & 34.6 & 100.0 \\
\hline (8) Immunology & 1.8 & 1.6 & 1.1 & 1.5 & 36.3 & 35.0 & 28.7 & 100.0 \\
\hline Physical sciences & 21.8 & 29.0 & 35.1 & 29.3 & 21.6 & 32.0 & 46.4 & 100.0 \\
\hline (9) Chemistry & 7.8 & 11.6 & 15.6 & 12.1 & 18.7 & 31.1 & 50.2 & 100.0 \\
\hline (10) Physics & 7.7 & 10.7 & 13.5 & 10.9 & 20.4 & 31.7 & 47.9 & 100.0 \\
\hline (11) Computer science & 2.1 & 2.0 & 1.8 & 2.0 & 31.1 & 33.9 & 35.0 & 100.0 \\
\hline (12) Mathematics & 2.4 & 2.8 & 2.9 & 2.7 & 25.7 & 33.6 & 40.6 & 100.0 \\
\hline (13) Space science & 1.8 & 1.8 & 1.4 & 1.6 & 32.4 & 35.6 & 32.0 & 100.0 \\
\hline Other natural sciences & 22.3 & 23.6 & 29.4 & 25.5 & 25.4 & 29.9 & 44.7 & 100.0 \\
\hline (14) Engineering & 7.1 & 6.8 & 8.9 & 7.7 & 26.7 & 28.7 & 44.6 & 100.0 \\
\hline (15) Plant and animal science & 5.4 & 5.5 & 6.6 & 5.9 & 26.4 & 30.3 & 43.3 & 100.0 \\
\hline (16) Materials science & 2.6 & 3.9 & 6.0 & 4.3 & 17.3 & 29.2 & 53.5 & 100.0 \\
\hline (17) Geoscience & 2.8 & 2.8 & 2.9 & 2.8 & 28.3 & 31.8 & 39.9 & 100.0 \\
\hline (18) Environment and ecology & 2.7 & 2.4 & 2.3 & 2.4 & 31.8 & 31.4 & 36.8 & 100.0 \\
\hline (19) Agricultural sciences & 1.4 & 1.9 & 2.0 & 1.8 & 22.5 & 33.8 & 43.6 & 100.0 \\
\hline (20) Multidisciplinary & 0.4 & 0.3 & 0.7 & 0.5 & 23.7 & 18.8 & 57.5 & 100.0 \\
\hline SOCIAL SCIENCES & 8.9 & 3.9 & 2.9 & 4.9 & 51.9 & 25.7 & 22.4 & 100.0 \\
\hline (21) Social sciences, general & 6.6 & 2.6 & 2.0 & 3.5 & 53.8 & 24.0 & 22.3 & 100.0 \\
\hline (22) Economics and business & 2.3 & 1.3 & 0.8 & 1.4 & 47.2 & 30.0 & 22.8 & 100.0 \\
\hline All sciences & 100.0 & 100.0 & 100.0 & 100.0 & 29.0 & 32.3 & 38.7 & 100.0 \\
\hline
\end{tabular}

Table 3

Critical citation levels (CCLs).

\begin{tabular}{lll}
$\mathrm{CCL}=$ 80th Percentile & \\
\hline $\mathrm{CCL}$ & $\begin{array}{l}\text { Multiple of the } \\
\text { mean }\end{array}$ & $\begin{array}{l}\text { Percentage of citations } \\
\text { among high-impact articles }\end{array}$ \\
(1) $(2)$ & (3)
\end{tabular}

$\mathrm{CCL}=95$ th Percentile

CCL Multiple of the Percentage of citations mean among high-impact articles (4) (5) (6)

$\begin{array}{lll}34 & 3.6 & 35.6 \\ 41 & 3.3 & 28.3 \\ 45 & 3.3 & 27.4 \\ 74 & 3.6 & 31.4 \\ 25 & 3.7 & 31.2 \\ 26 & 3.3 & 28.5 \\ 36 & 3.2 & 25.1 \\ 51 & 3.2 & 27.7\end{array}$

fe sciences

(1) Clinical medicine

$13 \quad 1.4$

69.0

(2) Biology and biochemistry

$\begin{array}{ll}19 & 1.5\end{array}$

60.2

(3) Neuroscience and behav. science

$20 \quad 1.5$

59.3

64.9

$\begin{array}{llll}\text { (5) Psychiatry and psychology } & 10 & 1.5 & 67.0\end{array}$

(6) Pharmacology and toxicology

60.7

(7) Microbiology

(8) Immunology

59.1

Physical sciences
(9) Chemistry
(10) Physics
(11) Computer science
(12) Mathematics
(13) Space science

$11 \quad 1.5$

65.5

69.1

79.2

$4 \quad 1.4$

$\begin{array}{ll}4 & 1.7\end{array}$

70.2

66.4

Other natural sciences
(14) Engineering
(15) Plant and animal science
(16) Materials science
(17) Geosciences
(18) Environment and ecology
(19) Agricultural sciences
(20) Multidisciplinary

$\begin{array}{lll}16 & 1.5 & 66.4\end{array}$

$5 \quad 1.6 \quad 70.6$

63.6

73.0

64.6

59.6

68.1

80.7

$\begin{array}{rrr}26 & 3.5 & 29.5 \\ 26 & 3.8 & 35.9 \\ 11 & 3.7 & 44.5 \\ 9 & 3.8 & 35.0 \\ 38 & 3.5 & 32.0\end{array}$

SOCIAL SCIENCES

(21) Social sciences, general

(22) Economics and business
70.5

69.2 $\begin{array}{lll}12 & 3.9 & 34.0\end{array}$

$\begin{array}{lll}17 & 3.4 & 31.4\end{array}$

$\begin{array}{lll}16 & 3.7 & 34.9\end{array}$

$\begin{array}{lll}23 & 3.5 & 27.6\end{array}$

$\begin{array}{lll}23 & 3.4 & 26.5\end{array}$

$\begin{array}{lll}16 & 3.4 & 31.1\end{array}$

$\begin{array}{lll}16 & 4.2 & 49.1\end{array}$

$\begin{array}{lll}5 & 1.6 & 69.2\end{array}$

$\begin{array}{ll}12 & 3.7\end{array}$

34.2

$\begin{array}{ll}15 & 3.9\end{array}$

34.9 
In most fields the number of citations corresponding to the 80th percentile is rather low (column 1): equal to or smaller than eight in nine cases, and from 10 to 13 in seven other fields, with a maximum of 29 citations for Molecular Biology and Genetics. However, the considerable differences in citation practices across fields clearly reveal themselves when the $95^{\text {th }}$ percentile is reached (column 4): among the Social, Physical and Other Natural Sciences the CCL varies from nine to 38 citations, while in eight Life Sciences the range goes from 25 to 74 . The maximum of 74 in Molecular Biology and Genetics is more than eight times greater than the minimum of nine citations in Mathematics.

Interestingly, the range of variation of the number of citations when the CCL is fixed at the $95^{\text {th }}$ percentile is dramatically reduced after normalization by the MCR (column 5). This is a consequence of the fact that, although the scale of the distribution - measured, for example, by a sufficiently large citation percentile or the MCR - is very different across sciences, the shape of the distribution is very similar indeed. This is confirmed by the relatively reduced range of variation of the (very high) percentages of total citations above the 80th or the 95th percentiles (columns 3 and 6). ${ }^{4}$

Of course, the fact that - with some exceptions - the shapes of the 22 citation distributions under study share many common features, tells us nothing in advance about how similar or different are the shapes of the three geographical areas' citation distributions under study when their high- and low-impact aspects are measured by the FGT indices presented in Section 2. This is the question investigated in the next section.

\section{Empirical results}

This section requires the computation of a rather large number of indicators. Consider the partition of the original number of articles in each field in seven subgroups, one for each type of authorship (as in Table 1). For each subgroup, the following six statistics are needed: three values for the members of the FGT high-impact family corresponding to $\beta=0,1$, and 2 , and three values for the corresponding members of the FGT low-impact family. Two overall, aggregate indicators must be also included. The first, which will be used in Section 4.4, is simply the sum of the seven subgroups' indicators weighted by the subgroups' publication shares. The second overall indicator, which will be used in Section 4.1, is somewhat more complex because it is the one that aggregates the indices for the three geographical areas in the extended count. These seven indicators for each field, as well as the two overall ones, are presented in Table A in the Appendix where the construction of the last two is explained in detail.

\subsection{Incidence, intensity, and citation inequality according to FGT indicators}

As indicated in Section 1, the first research question in this paper is the study of the consequences of measuring only the incidence, or also the intensity and the inequality aspects of the high- and the low-impact characteristics of the different geographical areas. For this purpose, recall from Albarrán et al. (2010c) that the FGT family of high-impact indicators is decomposable in the sense that, given a $\operatorname{CCL} z$ and a given parameter value $\beta$, the overall high-impact measure for any field with citation distribution $\boldsymbol{x}$ can be expressed as

$$
H_{\beta}(\boldsymbol{x} ; z)=\sum_{k} \omega_{k} H_{\beta}\left(\boldsymbol{x}^{\boldsymbol{k}} ; z\right)
$$

where $H_{\beta}\left(\boldsymbol{x}^{\boldsymbol{k}} ; z\right)$ is the high-impact index value for area $k=\mathrm{U} . S ., \mathrm{EU}, \mathrm{RW}$, and $\omega_{k}$ is the area's publication share in the total extended number of articles in the field. Similarly, the overall low-impact measure can be expressed as

$$
L_{\beta}(\boldsymbol{x} ; z)=\sum_{k} \omega_{k} L_{\beta}\left(\boldsymbol{x}^{\boldsymbol{k}} ; z\right)
$$

where $L_{\beta}\left(\boldsymbol{x}^{k} ; z\right)$ is the low-impact index value for area $k$. To interpret the results below adequately, it is important to make explicit that, from a normative point of view, for any area $k$ it is preferable to have a high $H_{\beta}\left(\boldsymbol{x}^{k} ; z\right)$ and a low $L_{\beta}\left(\boldsymbol{x}^{k} ; z\right) .^{5}$

In order to quantify the relative situation of any area, it is convenient to refer to the ratio $\omega_{k} H_{\beta}\left(x^{k} ; z\right) / H_{\beta}(\boldsymbol{x} ; z)$ as area $k$ 's observed contribution (OC hereafter) relative to the overall high-impact level for that $\beta$. We may ask: what is this area's relative expected contribution (EC hereafter) to that level? Clearly, its publication share $\omega_{k}$. Thus, the ratio OC/EC $=H_{\beta}\left(\boldsymbol{x}^{k}\right.$; $z) / H_{\beta}(\boldsymbol{x} ; z)$ is greater than, equal to, or smaller than one as area $k$ OC is greater than, equal to, or smaller than area $k$ EC. Similarly, the ratio $L_{\beta}\left(\boldsymbol{x}^{k} ; z\right) / L_{\beta}(\boldsymbol{x} ; z)$ is greater than, equal to, or smaller than one as area $k$ OC is greater than, equal to, or smaller than area $k$ EC. On the other hand, for any field's citation distribution $\boldsymbol{x}$, any CCL $z$, any $\beta$, and any two geographical

\footnotetext{
${ }^{4}$ Using the characteristic scores technique that serves to classify ordered citation distributions into several citation categories independently of differences in the MCR, in Schubert, Glänzel, and Braun (1987), Glänzel (2007), Albarrán and Ruiz-Castillo (2009), and Albarrán, Crespo, Ortuño, and Ruiz-Castillo (2010b) we learn how similar the percentage distributions of broad scientific fields and sub-fields are.

${ }^{5}$ Note that the publication shares $\omega_{k}$ for all fields are in columns 5-7 in Table 2, while the indices $H_{\beta}(\boldsymbol{x} ; z)$ and $L_{\beta}(\boldsymbol{x} ; z)$ for all $\beta$, all $\boldsymbol{x}$, and a CCL $z$ equal to the 80th percentile of the world citation distribution in any field, appear in column 9 in Appendix A.
} 
The ratio of observed contributions (OC) to high-impact overall levels to expected contributions (EC) By geographical area in every scientific field ${ }^{\text {a }}$ the OC captures only the incidence $(\beta=0)$, the incidence and the intensity $(\beta=1)$, or the incidence, the intensity, and the inequality $(\beta=2)$ of the high-impact phenomenon.

\begin{tabular}{|c|c|c|c|c|c|c|c|c|c|}
\hline & \multicolumn{3}{|c|}{ United States } & \multicolumn{3}{|c|}{ European Union } & \multicolumn{3}{|c|}{ Rest of the world } \\
\hline & $\begin{array}{l}\beta=0 \\
(1)\end{array}$ & $\begin{array}{l}\beta=1 \\
(2)\end{array}$ & $\begin{array}{l}\beta=2 \\
(3)\end{array}$ & $\begin{array}{l}\beta=0 \\
(4)\end{array}$ & $\begin{array}{l}\beta=1 \\
(5)\end{array}$ & $\begin{array}{l}\beta=2 \\
(6)\end{array}$ & $\begin{array}{l}\beta=0 \\
(7)\end{array}$ & $\begin{array}{l}\beta=1 \\
(8)\end{array}$ & $\begin{array}{l}\beta=2 \\
(9)\end{array}$ \\
\hline \multicolumn{10}{|l|}{ Life sciences } \\
\hline (1) Clinical medicine & 1.30 & 1.42 & 1.55 & 0.95 & 0.89 & 0.80 & 0.75 & 0.69 & 0.66 \\
\hline (2) Biology and biochemistry & 1.45 & 1.56 & 1.73 & 0.95 & 0.90 & 0.86 & 0.65 & 0.60 & 0.49 \\
\hline (3) Neuroscience and behav. sci. & 1.32 & 1.45 & 1.58 & 0.94 & 0.87 & 0.78 & 0.68 & 0.61 & 0.54 \\
\hline (4) Molecular biology and genetics & 1.30 & 1.39 & 1.48 & 0.95 & 0.89 & 0.81 & 0.67 & 0.63 & 0.60 \\
\hline (5) Psychiatry and psychology & 1.12 & 1.20 & 1.31 & 0.95 & 0.88 & 0.73 & 0.79 & 0.70 & 0.64 \\
\hline (6) Pharmacology and toxicology & 1.42 & 1.55 & 1.57 & 1.05 & 1.04 & 1.11 & 0.69 & 0.61 & 0.55 \\
\hline (7) Microbiology & 1.43 & 1.58 & 1.75 & 1.01 & 0.96 & 0.88 & 0.63 & 0.56 & 0.51 \\
\hline (8) Immunology & 1.26 & 1.32 & 1.34 & 0.92 & 0.87 & 0.74 & 0.76 & 0.76 & 0.88 \\
\hline \multicolumn{10}{|l|}{ PHYSICAL SCIENCES } \\
\hline (9) Chemistry & 1.60 & 1.96 & 1.99 & 1.13 & 1.04 & 1.16 & 0.70 & 0.61 & 0.53 \\
\hline (10) Physics & 1.46 & 1.71 & 2.08 & 1.13 & 1.09 & 0.96 & 0.72 & 0.64 & 0.57 \\
\hline (11) Computer science & 1.31 & 1.53 & 1.61 & 0.95 & 0.87 & 0.53 & 0.78 & 0.66 & 0.92 \\
\hline (12) Mathematics & 1.25 & 1.47 & 1.97 & 1.10 & 1.04 & 0.81 & 0.76 & 0.66 & 0.54 \\
\hline (13) Space science & 1.34 & 1.39 & 1.39 & 0.97 & 0.96 & 0.98 & 0.68 & 0.65 & 0.63 \\
\hline \multicolumn{10}{|l|}{ Other natural sciences } \\
\hline (14) Engineering & 1.21 & 1.37 & 1.62 & 1.11 & 1.09 & 1.04 & 0.81 & 0.72 & 0.61 \\
\hline (15) Plant and animal science & 1.21 & 1.33 & 1.57 & 1.18 & 1.19 & 1.07 & 0.75 & 0.67 & 0.60 \\
\hline (16) Materials science & 1.37 & 1.74 & 2.27 & 1.12 & 1.05 & 0.94 & 0.82 & 0.73 & 0.62 \\
\hline (17) Geoscience & 1.37 & 1.49 & 1.55 & 1.06 & 1.00 & 0.98 & 0.69 & 0.65 & 0.63 \\
\hline (18) Environment and ecology & 1.21 & 1.32 & 1.52 & 1.06 & 1.01 & 0.94 & 0.77 & 0.71 & 0.60 \\
\hline (19) Agricultural sciences & 1.27 & 1.47 & 1.67 & 1.21 & 1.18 & 1.17 & 0.70 & 0.62 & 0.52 \\
\hline (20) Multidisciplinary & 1.89 & 2.25 & 2.52 & 1.32 & 1.32 & 1.12 & 0.53 & 0.38 & 0.33 \\
\hline \multicolumn{10}{|l|}{ Social sciences } \\
\hline (21) Social sciences, general & 1.13 & 1.22 & 1.33 & 0.95 & 0.86 & 0.74 & 0.75 & 0.62 & 0.49 \\
\hline (22) Economics and business & 1.30 & 1.43 & 1.50 & 0.81 & 0.69 & 0.62 & 0.64 & 0.53 & 0.46 \\
\hline
\end{tabular}

In row (1) Clinical Medicine, for example, when only the incidence of the high-impact phenomenon is taken into account: (i) the U.S. OC according to $H_{0}$ is $30 \%$ above the corresponding EC; (ii) the EU OC according to $H_{0}$ is $5 \%$ below the corresponding EC, and (iii) the RW OC according to that index is $25 \%$ below the corresponding EC. When the aggregate gap between the citations received by high-impact articles and the CCL is taken into account, the U.S. OC according to $H_{1}$ is $42 \%$ above the EC. Finally, when the citation inequality among high-impact articles is also taken into account, the U.S. OC according to $\mathrm{H}_{2}$ is $55 \%$ above its EC.

a In any field and any column, a cell value is greater than, equal to, or smaller than one when the geographical area's observed contribution (OC) to the world high-impact level is greater than, equal to, or smaller than the area's publication share (or expected contribution, EC) in the extended citation distribution.

areas $k$ and $l$, it is obvious that

$$
\begin{aligned}
& \frac{H_{\beta}\left(\boldsymbol{x}^{\boldsymbol{k}} ; z\right)}{H_{\beta}(\boldsymbol{x} ; z)}>\frac{H_{\beta}\left(\boldsymbol{x}^{l} ; z\right)}{H_{\beta}(\boldsymbol{x} ; z)} \Rightarrow H_{\beta}\left(\boldsymbol{x}^{\boldsymbol{k}} ; z\right)>H_{\beta}\left(\boldsymbol{x}^{l} ; z\right) \\
& \text { and } \\
& \frac{L_{\beta}\left(\boldsymbol{x}^{\boldsymbol{k}} ; z\right)}{L_{\beta}(\boldsymbol{x} ; z)}>\frac{L_{\beta}\left(\boldsymbol{x}^{l} ; z\right)}{L_{\beta}(\boldsymbol{x} ; z)} \Rightarrow L_{\beta}\left(\boldsymbol{x}^{\boldsymbol{k}} ; z\right)>L_{\beta}\left(\boldsymbol{x}^{l} ; z\right) .
\end{aligned}
$$

The information about the ratios $H_{\beta}\left(\boldsymbol{x}^{k} ; z\right) / H_{\beta}(\boldsymbol{x} ; z)$ and $L_{\beta}\left(\boldsymbol{x}^{k} ; z\right) / L_{\beta}(\boldsymbol{x} ; z)$ for every $k$, every field, and every $\beta$ in the two FGT families of high- and low-impact indicators is in Tables $4 \mathrm{~A}$ and $4 \mathrm{~B}$, where the meaning of the numbers is explained. The results are illustrated in Figs. 1 and 2, where the left bars are for the U.S., the middle bars for the EU, and the right bars for the RW. In each field and each area, the three bars in Figure 1 reflect the ratios $H_{\beta}\left(\boldsymbol{x}^{k} ; z\right) / H_{\beta}(\boldsymbol{x} ; z)$ for $\beta=0,1$, and 2 . Similarly, the three bars in Fig. 2 reflect the ratios $L_{\beta}\left(\boldsymbol{x}^{k} ; z\right) / L_{\beta}(\boldsymbol{x} ; z)$ for $\beta=0,1$, and 2 .

\subsubsection{High- and low-impact rankings}

As pointed out in the notes to Table 4A, in Clinical Medicine the ratio OC/EC is strictly greater in the U.S. than in the $\mathrm{EU}$, and in the latter than in the RW when $\beta=0$. Hence, by expression (3) in Section 4.1 it can be concluded that in Clinical Medicine the high-impact level according to $H_{0}$ is strictly greater in the U.S. than in the EU, and in the latter than in the RW. As a matter of fact, this is the same ranking that is observed in Table 4A and Fig. 1 for all fields and all values of $\beta$ (with the exception of Immunology, where the high-impact level according to $\mathrm{H}_{2}$ is strictly smaller in the EU than in the RW). Similarly, in Table 4B and Fig. 2 it is observed that the low-impact level according to all indicators is strictly lower in the U.S. than in the EU, and in the later than in the RW (except in Engineering, where the low-impact level according to $L_{1}$ and $L_{2}$ 
The ratio of observed contributions (OC) to low-impact overall levels to expected contributions (EC) by geographical area in every scientific field ${ }^{\mathrm{a}}$ the OC captures only the incidence $(\beta=0)$, the incidence and the intensity $(\beta=1)$, or the incidence, the intensity, and the inequality ( $\beta=2)$ of the low-impact phenomenon.

\begin{tabular}{|c|c|c|c|c|c|c|c|c|c|}
\hline & \multicolumn{3}{|c|}{ United States } & \multicolumn{3}{|c|}{ European Union } & \multicolumn{3}{|c|}{ Rest of the world } \\
\hline & $\begin{array}{l}\beta=0 \\
(1)\end{array}$ & $\begin{array}{l}\beta=1 \\
(2)\end{array}$ & $\begin{array}{l}\beta=2 \\
(3)\end{array}$ & $\begin{array}{l}\beta=0 \\
(4)\end{array}$ & $\begin{array}{l}\beta=1 \\
(5)\end{array}$ & $\begin{array}{l}\beta=2 \\
(6)\end{array}$ & $\begin{array}{l}\beta=0 \\
(7)\end{array}$ & $\begin{array}{l}\beta=1 \\
(8)\end{array}$ & $\begin{array}{l}\beta=2 \\
(9)\end{array}$ \\
\hline \multicolumn{10}{|l|}{ Life sciences } \\
\hline (1) Clinical medicine & 0.92 & 0.87 & 0.84 & 1.01 & 1.03 & 1.04 & 1.07 & 1.11 & 1.13 \\
\hline (2) Biology and biochemistry & 0.89 & 0.80 & 0.75 & 1.01 & 0.99 & 0.98 & 1.08 & 1.18 & 1.24 \\
\hline (3) Neuroscience and behav. sci. & 0.92 & 0.86 & 0.82 & 1.01 & 1.02 & 1.02 & 1.08 & 1.16 & 1.20 \\
\hline (4) Molecular biology and genetics & 0.92 & 0.85 & 0.82 & 1.01 & 1.00 & 0.99 & 1.09 & 1.19 & 1.24 \\
\hline (5) Psychiatry and psychology & 0.97 & 0.94 & 0.93 & 1.01 & 1.03 & 1.04 & 1.05 & 1.10 & 1.12 \\
\hline (6) Pharmacology and toxicology & 0.90 & 0.86 & 0.84 & 0.99 & 0.97 & 0.96 & 1.07 & 1.11 & 1.13 \\
\hline (7) Microbiology & 0.89 & 0.80 & 0.75 & 1.00 & 0.96 & 0.94 & 1.10 & 1.21 & 1.27 \\
\hline (8) Immunology & 0.93 & 0.87 & 0.83 & 1.02 & 1.03 & 1.04 & 1.06 & 1.13 & 1.16 \\
\hline \multicolumn{10}{|l|}{ Physical sciences } \\
\hline (9) Chemistry & 0.85 & 0.76 & 0.72 & 0.97 & 0.90 & 0.85 & 1.08 & 1.15 & 1.19 \\
\hline (10) Physics & 0.89 & 0.83 & 0.80 & 0.97 & 0.93 & 0.92 & 1.07 & 1.12 & 1.14 \\
\hline (11) Computer science & 0.93 & 0.90 & 0.89 & 1.01 & 1.01 & 1.01 & 1.05 & 1.08 & 1.09 \\
\hline (12) Mathematics & 0.95 & 0.91 & 0.89 & 0.98 & 0.95 & 0.93 & 1.05 & 1.10 & 1.13 \\
\hline (13) Space science & 0.90 & 0.82 & 0.77 & 1.01 & 1.01 & 1.02 & 1.09 & 1.17 & 1.21 \\
\hline \multicolumn{10}{|l|}{ Other natural sciences } \\
\hline (14) Engineering & 0.96 & 0.94 & 0.94 & 0.98 & 0.94 & 0.93 & 1.04 & 1.07 & 1.08 \\
\hline (15) Plant and animal science & 0.95 & 0.91 & 0.89 & 0.96 & 0.92 & 0.90 & 1.06 & 1.11 & 1.14 \\
\hline (16) Materials science & 0.91 & 0.86 & 0.84 & 0.97 & 0.94 & 0.92 & 1.05 & 1.08 & 1.09 \\
\hline (17) Geoscience & 0.90 & 0.82 & 0.79 & 0.98 & 0.93 & 0.90 & 1.08 & 1.18 & 1.23 \\
\hline (18) Environment and ecology & 0.95 & 0.93 & 0.92 & 0.99 & 0.95 & 0.93 & 1.05 & 1.11 & 1.13 \\
\hline (19) Agricultural sciences & 0.93 & 0.87 & 0.83 & 0.95 & 0.89 & 0.86 & 1.08 & 1.16 & 1.20 \\
\hline (20) Multidisciplinary & 0.80 & 0.72 & 0.69 & 0.93 & 0.91 & 0.91 & 1.11 & 1.14 & 1.16 \\
\hline \multicolumn{10}{|l|}{ Social sciences } \\
\hline (21) Social sciences, general & 0.97 & 0.96 & 0.95 & 1.01 & 1.00 & 1.00 & 1.06 & 1.09 & 1.11 \\
\hline (22) Economics and business & 0.94 & 0.90 & 0.89 & 1.04 & 1.05 & 1.06 & 1.08 & 1.13 & 1.16 \\
\hline
\end{tabular}

In row (1) Clinical Medicine, for example, when only the incidence of the low-impact phenomenon is taken into account it can be seen that the U.S., the EU, and the RW OC according to $L_{0}$ is $8 \%$ below, $1 \%$ above, and $7 \%$ above the corresponding EC, respectively.

a In any field and any column, a cell value is greater than, equal to, or smaller than one when the geographical area's observed contribution (OC) to the world low-impact level is greater than, equal to, or smaller than the area's publication share (or expected contribution, EC) in the extended citation distribution.

in the U.S. is equal to or strictly greater than in the EU). ${ }^{6}$ These results show the overwhelming superiority of the U.S. in all sciences from an ordinal point of view.

\subsubsection{High- and low-impact cardinal comparisons}

Coming now to the cardinal aspects, the question is: does the relative situation of the geographical areas differ when they are evaluated according to different members of the FGT families? For a detailed answer, the reader must consult Tables $4 \mathrm{~A}$ and $4 \mathrm{~B}$, while Figs. 1 and 2 facilitate a qualitative illustration.

Starting with high-impact levels and the U.S. case, as parameter $\beta$ takes values 0,1 , and 2 the ratio OC/EC strictly increases in 21 fields, and remains constant in the remaining case (Space Science for $\beta=1,2$ ). These increases are considerable (columns 1-3 in Table 4A): at a minimum, in Immunology and Space Science it is of five or eight percentage points, while in Materials Science, the Multidisciplinary field, Mathematics, and Physics, the increases are between 62 and 80 percentage points. All in all, the U.S. occupies a dominant position in the world in all fields: when $\beta=2$ (column 3 in Table 4A), the ratio OC/EC goes from a low 33-39\% (in Social Sciences, Immunology, and Space Science) to a high 97-154\% (in Mathematics, Chemistry, Materials Science, and the Multidisciplinary field). Essentially, as can be seen in columns 7-9 in Table 4A and Fig. 1, the RW presents the opposite pattern: the ratio OC/EC systematically decreases as $\beta$ increases except in Immunology and Computer Science. In the latter field, the $\mathrm{OC}$ is $8 \%$ below the EC according to $\mathrm{H}_{2}$. This is the RW's most favorable case, since the OC according to $\mathrm{H}_{2}$ is from $36 \%$ to $67 \%$ below the corresponding EC in 20 disciplines (column 9 in Table 4A).

Finally, the EU high-impact performance is not very impressive. The OC/EC ratio in the EU continuously decreases as $\beta$ increases in 16 cases, including six in which that ratio starts being above one when $\beta=0$ to being below one when $\beta=2$ (Microbiology, Physics, Mathematics, Materials Science, Geosciences, and Environment and Ecology). Thus, when the incidence, intensity, and inequality aspects are taken into account (column 6 in Table 4A), the OC/EC ratio according to $\mathrm{H}_{2}$

\footnotetext{
${ }^{6}$ These two exceptions indicate that to establish a ranking, even among only three areas, distributional considerations incorporated into $\mathrm{H}_{2}$ and $L_{2}$ can make a difference.
} 
in the EU is well below one in many fields (Materials Science, Geosciences, and Environment and Ecology, the two Social Sciences, all Physical Sciences except Chemistry, and all Life Sciences except Pharmacology and Toxicology). This means, of course, that the high-impact level of the EU in those fields is below what can be expected from its publication share. This ratio is above one in four cases (Engineering, Plant and Animal Science, Multidisciplinary, and Pharmacology and Toxicology), and considerably greater than one only in Agricultural Sciences and Chemistry. These two fields provide a good example of the subtleties captured in this approach. According to $H_{0}$ (column 4 in Table 4A), Agricultural Sciences in the EU do rather better than Chemistry. This is exactly what we found with J. Crespo in Albarrán, Crespo, Ortuño, \& Ruiz-Castillo (2010a) where only incidence aspects are taken into account. However, when the intensity and inequality dimensions are incorporated in $\mathrm{H}_{2}$ Chemistry comes practically equal to Agricultural Sciences (column 6 in Table 4A).

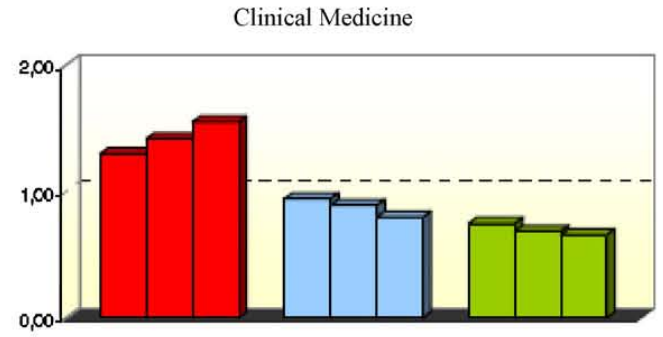

Neuroscience \& Behaviour Science

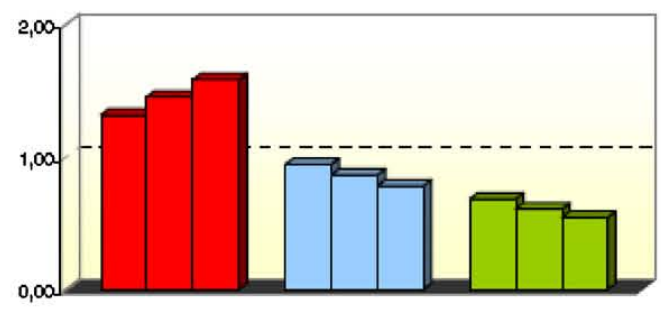

Psychiatry \& Psychology

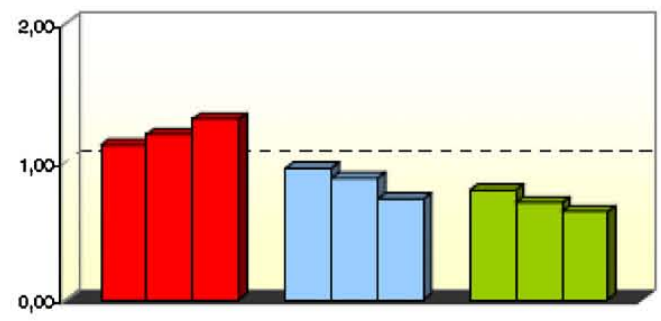

Microbiology

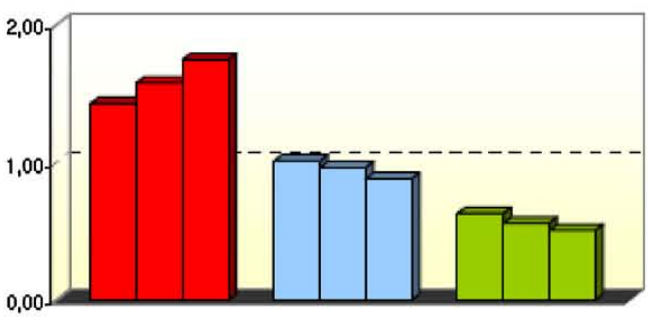

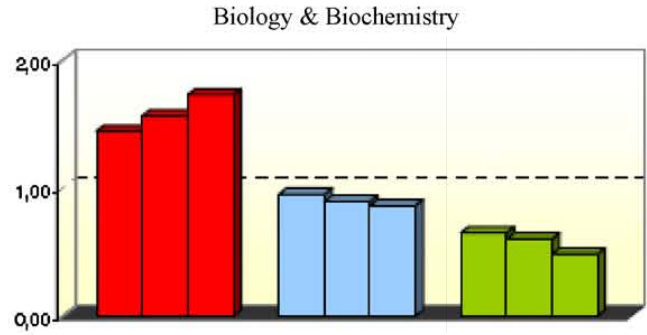

Molecular Biology \& Genetics

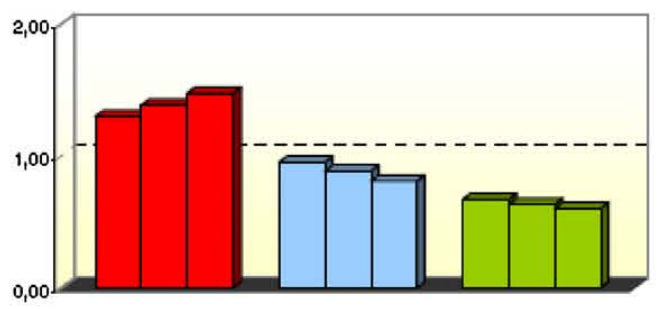

Pharmacology \& Toxicology

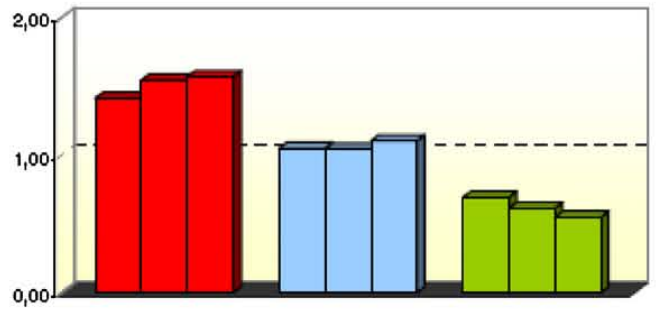

Immunology

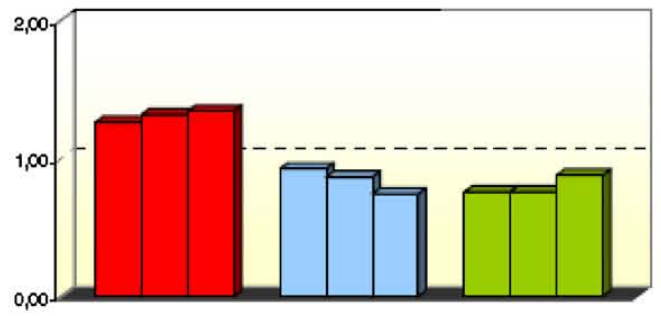

Fig. 1. The relative contribution to world high-impact levels by the U.S. (left bars), the EU (middle bars), and the RW (right bars) according to incidence, intensity, and citation inequality members of the FGT family of high-impact indicators. 
Chemistry

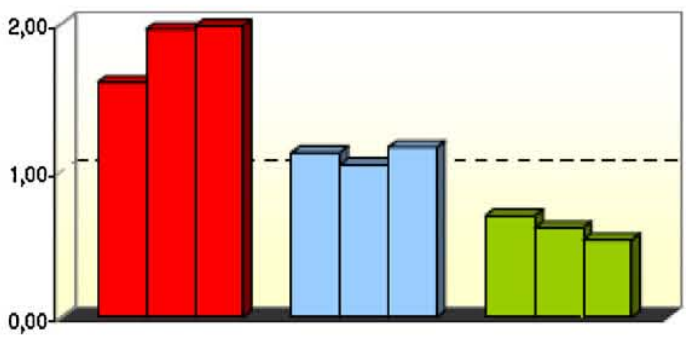

Computer Science

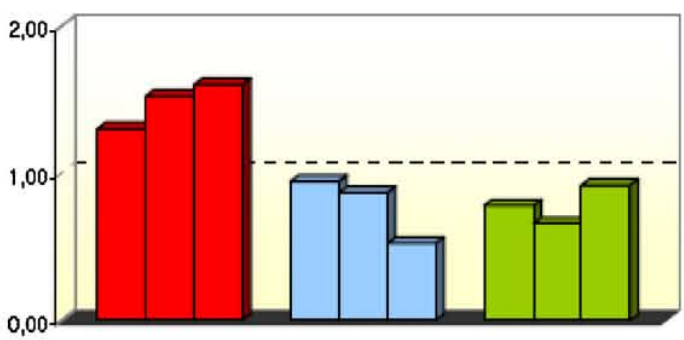

Space Science

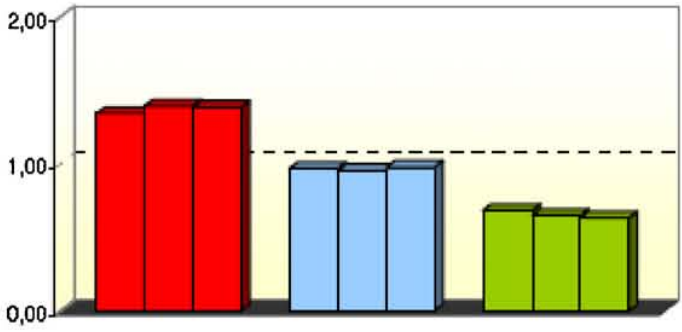

Plant \& Animal Science

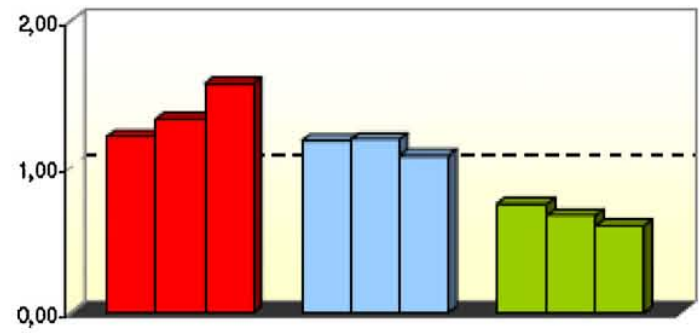

Physics

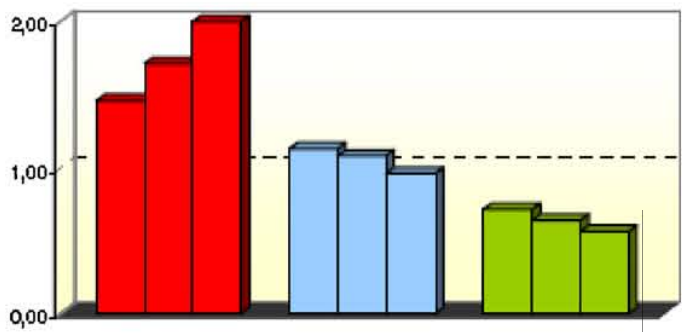

Mathematics

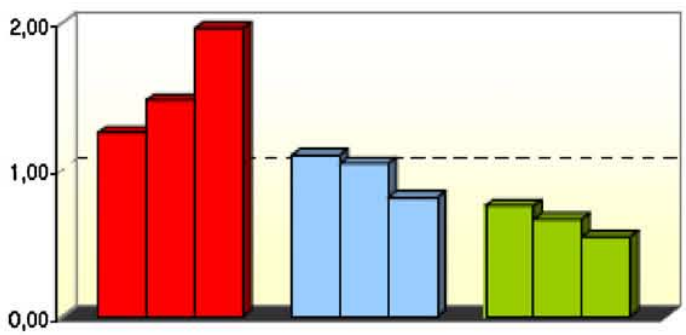

Engineering

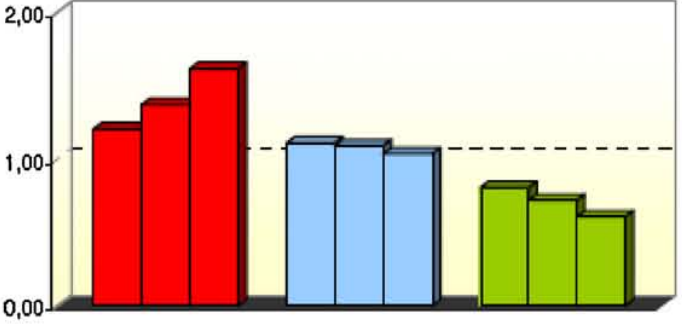

Materials Science

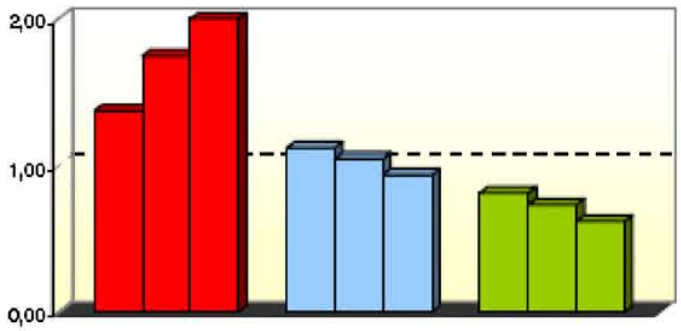

Fig. 1. (Continued)

As far as low-impact levels is concerned, the U.S. contribution to overall low-impact levels in Clinical Medicine according to $L_{0}, L_{1}$, and $L_{2}$ is $8 \%, 13 \%$, and $16 \%$ below what could be expected from its publication share (columns $1-3$ in Table $4 \mathrm{~B}$ ). As can be clearly seen in Fig. 2, this is again a general pattern that clearly speaks about the U.S. good scientific performance: when the CCL is fixed at the 80th percentile of the world original distribution, the U.S. OC/EC ratio is slightly below one in all sciences when only the incidence aspect is taken into account. But in all cases this ratio decreases, and hence the U.S. performance improves, as the intensity and the inequality aspects enter into the picture. When $\beta=2$ (column 3 in Table 4B), the U.S. OC according to $L_{2}$ is between $5 \%$ and $20 \%$ percentage points below the corresponding EC in 14 fields, and between $21 \%$ and $31 \%$ in six more fields (Geoscience, Space Science, Biology and Biochemistry, Microbiology, Chemistry, and the Multidisciplinary field). 
Geoscience

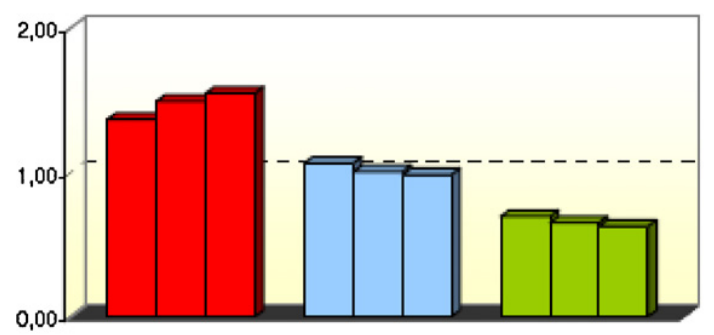

Agricultural Sciences

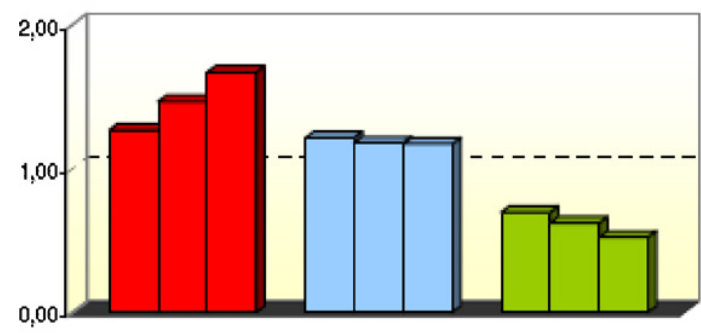

Social Sciences, General

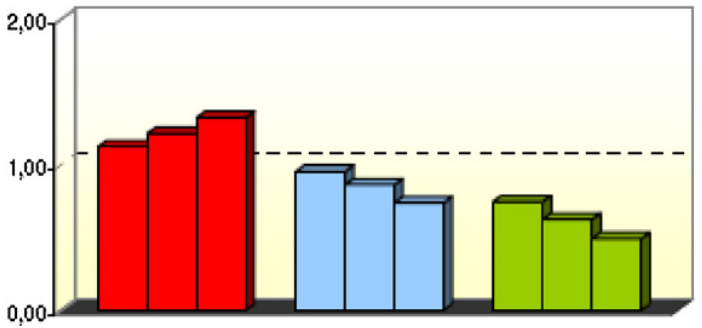

Environment \& Ecology

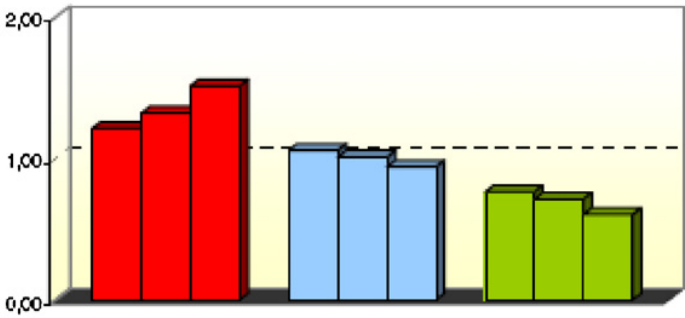

Multidisciplinary

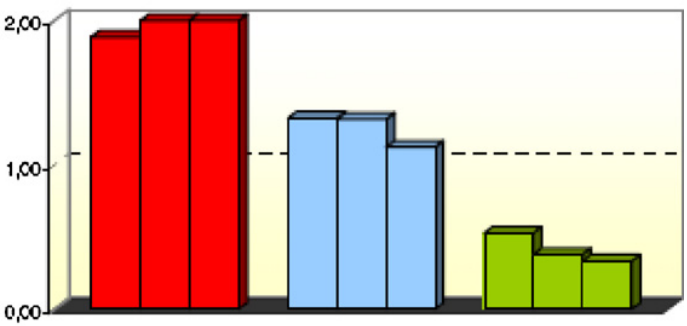

Economics \% Business

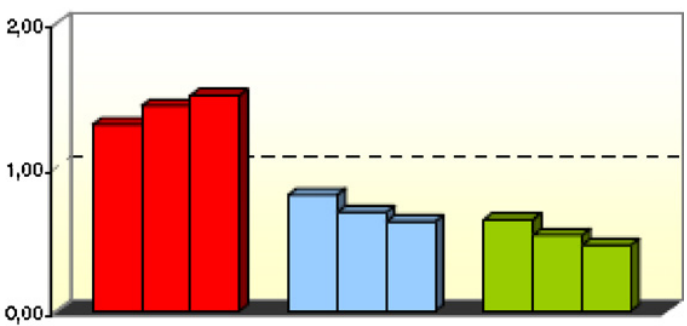

Fig. 1. (Continued).

As before, in the RW the situation is completely reversed. When only the incidence aspect is considered (column 7 in Table 4B), the OC is always four to 11 percentage points above the $\mathrm{EC}$, but when $\beta=2$ (column 9 in Table $4 \mathrm{~B}$ ) the OC/EC ratio is always greater than according to $L_{0}$, and in 11 cases the $\mathrm{OC}$ is about 16 percentage points above the EC. The EU presents mixed but moderate results. In 15 cases the OC/EC ratio decreases as $\beta$ increases, while in five cases it remains essentially constant. But in eight disciplines out of these 15 the changes in absolute value are below six percentage points. The OC/EC ratio when $\beta=2$ (column 6 in Table 4B) is greater than or equal to one in eight cases (including the two Social Sciences, Neuroscience and Behavioral Science, Psychology and Psychiatry, as well as Clinical Medicine and Immunology). On the bright side, the OC is at least seven percentage points below the EC in most Physical and Other Natural Sciences (except Computer Science, and Space Science), with Agricultural Sciences, and Chemistry on top.

In brief, considering only the percentages of high- and low-impact articles $(\beta=0)$, or adding up the aggregate citation gap between high-impact articles and the CCL or between the CCL and low-impact articles ( $\beta=1)$, or including the effect of distributional considerations $(\beta=2)$ generates important differences in all areas. Specifically, the relative scientific performance of the U.S. with respect to both the high- and the low-impact characteristics of citation distributions are essentially reinforced for all sciences as $\beta$ increases. The opposite is the case for the RW, while for the EU the results are more mixed: except for some important exceptions, the high-impact performance of the EU leaves much to be desired as $\beta$ increases, while the low-impact performance improves in a majority of cases but - with some exceptions also - by a reduced order of magnitude.

Therefore, in the rest of this section it would suffice to use the high- and low-impact indicators $\mathrm{H}_{2}$ and $L_{2}$ that simultaneously include the incidence, the intensity, and the inequality of the phenomena in question.

\subsection{Publication shares and high- and low-impact levels}

The previous sub-section established a clear high- and low-impact ranking - the U.S. above the EU, and the EU above the RW - independently of the member of the two FGT families used in the evaluation (with only two exceptions). However, the 
RW leads in publication share in 13 fields, the EU in three, and the U.S. in the remaining six (see columns 3-7 in Table 2). This contrast should serve to conclude without further statistical analysis that the connection for any geographical area between having a large publication share in a given field and a good index of high- or low-impact is practically non-existent.

A different matter is the relationship between the publication effort devoted to the various fields in each geographical area (columns 1-3 in Table 2) and the high- and low-impact levels achieved by this area across fields. In a linear model, the Pearson correlation coefficient between publication efforts and high-impact levels according to $\mathrm{H}_{2}$ is only -0.09 for the U.S., 0.11 for the EU, and essentially 0 for the RW. On the other hand, the Pearson correlation coefficient between publication efforts and low-impact levels according to $L_{2}$ is 0.05 for the U.S., 0 for the EU, and -0.18 for the RW. Thus, except perhaps for the weak negative association between publication effort and a good low-impact performance in the RW case, there is practically no connection between these variables.

The conclusion is inescapable: a large publication share by a geographical area in a given field does nor guarantee a good performance by this area in terms of a large high-impact level or a small low-impact index in that field. Similarly, a large publication effort in specific fields by any of the three large geographical areas does not guarantee a relatively good performance in those fields.

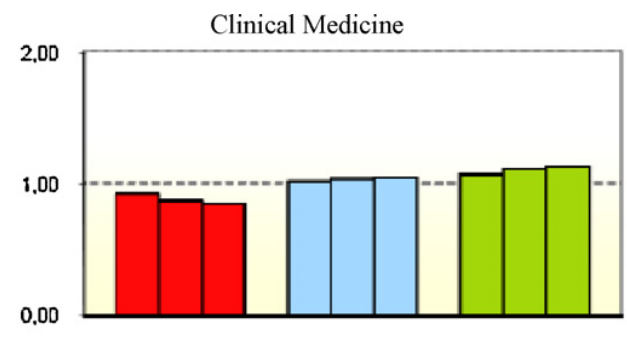

Neuroscience \& Behaviour Science

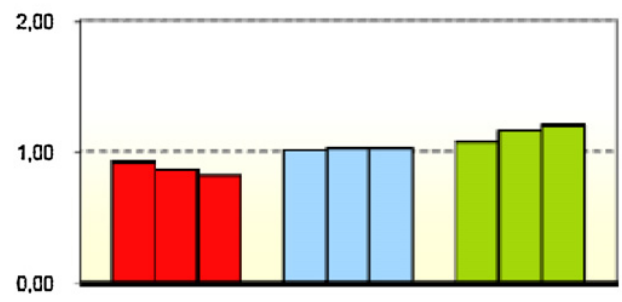

Psychiatry \& Psychology

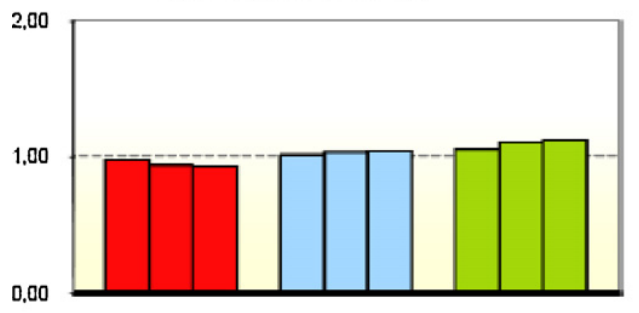

Microbiology

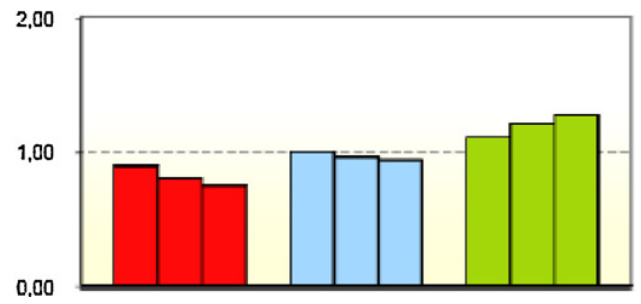

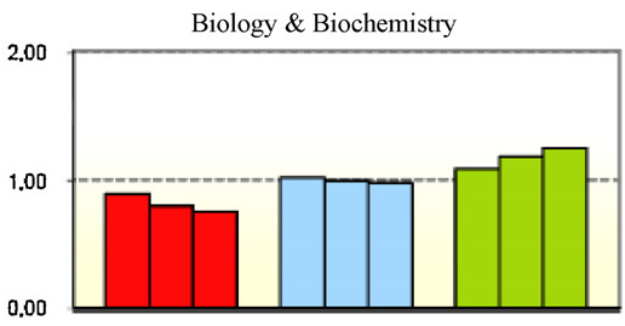

Molecular Biology \& Genetics

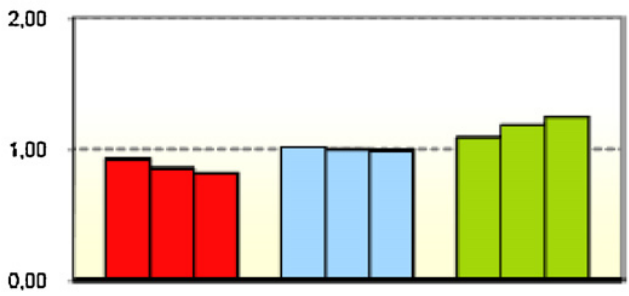

Pharmacology \& Toxicology

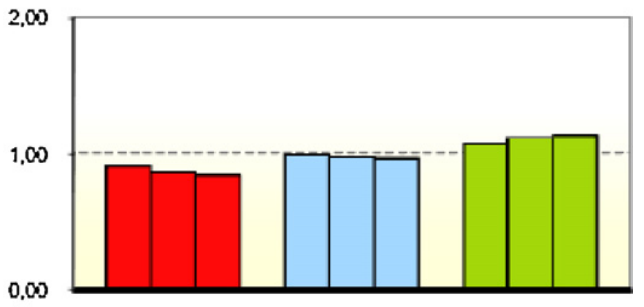

Immunology

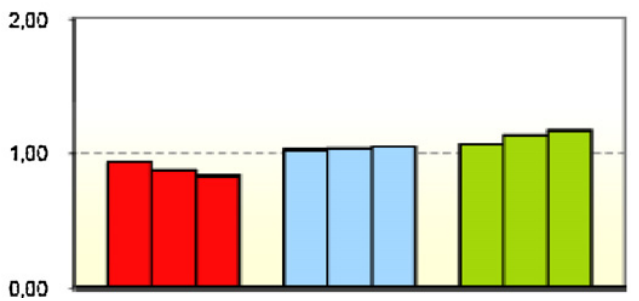

Fig. 2. The relative contribution to world low-impact levels by the U.S. (left bars), the EU (middle bars), and the rw (left bars) according to incidence, intensity, and citation inequality members of the FGT family of high-impact indicators. 
Chemistry

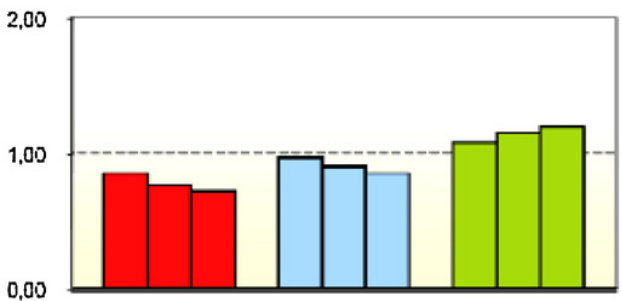

Computer Science

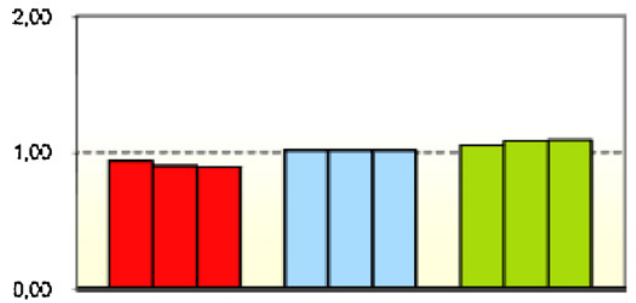

Space Science

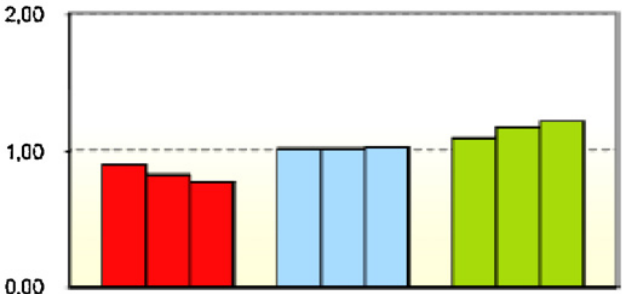

Engineering

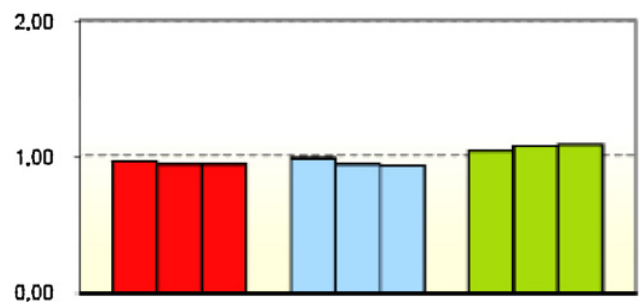

Physics

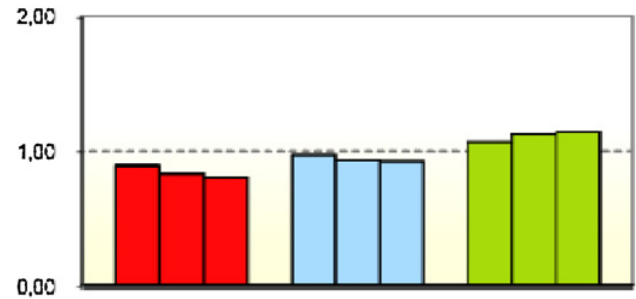

Mathematics

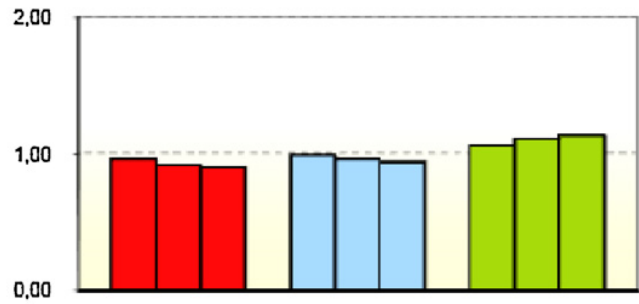

Fig. 2. (Continued)

\subsection{The effect of changes in the CCL}

The third research question is the study of how the relative situation of each area in each field according to $\mathrm{H}_{2}$ and $L_{2}$ varies when the CCL is increased. Table 5 provides the relevant information when the CCLs are fixed at the 80th and the 95th percentiles of the original citation distributions at the world level.

Let us begin with the consequences of the change in the CCL over the OC/EC ratios according to $\mathrm{H}_{2}$. One of the properties of the FGT families of high-impact indicators is that when the CCL is raised, say from $z$ to $z^{\prime}$, for any $\beta$ the high-impact level of any citation distribution necessarily increases; that is, $H_{\beta}(\boldsymbol{x} ; z)<H_{\beta}\left(\boldsymbol{x} ; z^{\prime}\right)$ for all $\boldsymbol{x}$ and $\beta$. However, for any particular area $k$ the relationship between the ratio $H_{2}\left(\boldsymbol{x}^{\boldsymbol{k}} ; z^{\prime}\right) / H_{2}\left(\boldsymbol{x} ; z^{\prime}\right)$ and $H_{2}\left(\boldsymbol{x}^{\boldsymbol{k}} ; z\right) / H_{2}(\boldsymbol{x} ; z)$ is an empirical question.

In the first place, it turns out that the relative situation of the U.S. improves in 17 cases, that is, the U.S. OC/EC ratio increases as the CCL is raised (compare columns 1 and 2 in Table 5). However, only in nine fields do these increases that take place through a worsening of the relative position of both the EU (columns 3 and 4) and the RW (columns 5 


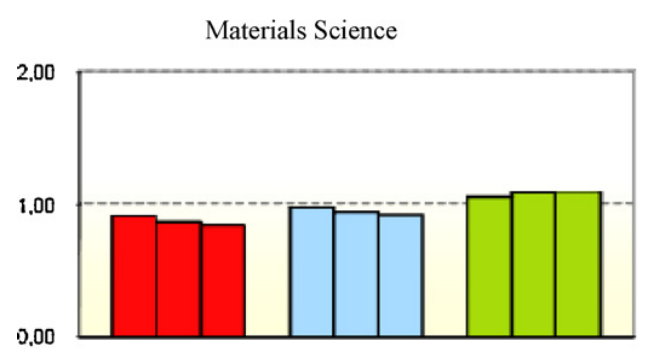

Environment \& Ecology

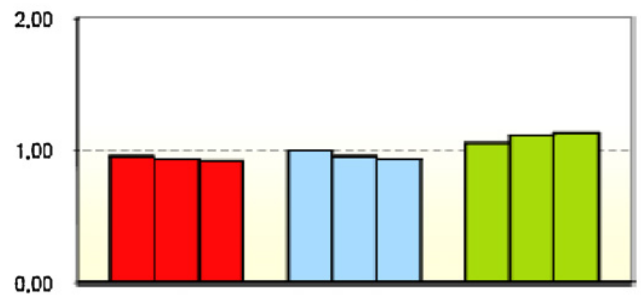

Multidisciplinary
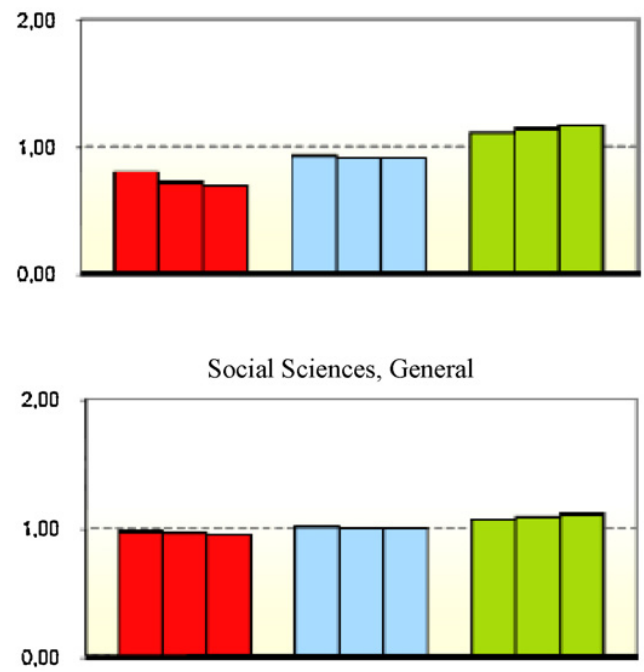
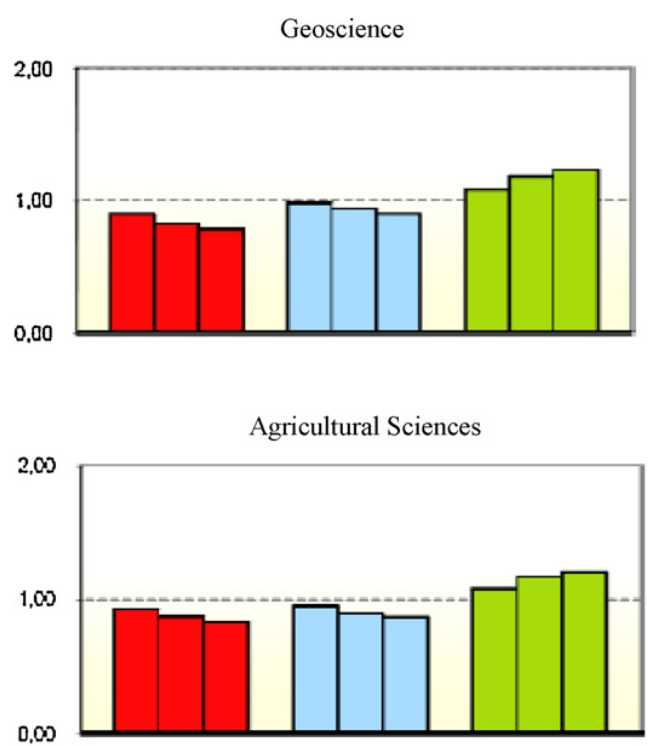

Fig. 2. (Continued).

and 6) - represent more than $15 \%$ of the level that the U.S. already achieves when the CCL is fixed at the $80^{\text {th }}$ percentile. In the second place, the situation in Immunology and Computer Science is quite exceptional. The OC/EC ratio for the U.S. remains essentially constant, while that of the EU is reduced. Therefore, this ratio for the RW increases as the CCL is raised. It should be emphasized that these are the only instances in which the RW scientific performance evolves so favorably since in all remaining fields the OC/EC ratio of the RW worsens or remains constant as the CCL increases. However, the order of magnitude of such reductions is again rather moderate (the worsening is below $10 \%$ of the level reached at the lower CCL, except in four fields where the RW's CO is already very low when the CCL is at the 80th percentile (Engineering, Environment and Ecology, Social Sciences, General, and Biology and Biochemistry). In the third place, in the other three cases in which the U.S. ratio worsens (Pharmacology and Toxicology, Space Science, and Chemistry), the beneficiary is the EU. This is a rather exceptional situation, since in only two other instances (Geosciences and Agricultural Sciences) does the EU ratio increase albeit by one percentage point - when the CCL is raised. In 15 fields the performance of the EU slightly worsens (by less than $10 \%$ ), or remains essentially constant. The deterioration of its OC/EC ratio above that percentage takes place in fields where the EU is already weak when the CCL is fixed at the 80th percentile (Microbiology, Psychology and Psychiatry, Mathematics and, as has already been pointed out, Immunology). 
Table 5

The relative contribution to high- and low-impact overall levels by geographical area in every scientific field when $\beta=2$ and the critical citation line (CCL) is equal to the 80 th and the 95 th percentile of the original citation distribution.

\begin{tabular}{|c|c|c|c|c|c|c|c|c|c|c|c|c|}
\hline \multirow[t]{3}{*}{ Percentile } & \multicolumn{6}{|c|}{ High-impact } & \multicolumn{6}{|c|}{ Low-impact } \\
\hline & \multicolumn{2}{|l|}{ U.S. } & \multicolumn{2}{|l|}{ EU } & \multicolumn{2}{|l|}{ RW } & \multicolumn{2}{|l|}{ U.S. } & \multicolumn{2}{|l|}{ EU } & \multicolumn{2}{|l|}{ RW } \\
\hline & $\begin{array}{l}\text { 95th } \\
\text { (1) }\end{array}$ & $\begin{array}{l}\text { 80th } \\
(2)\end{array}$ & $\begin{array}{l}\text { 95th } \\
\text { (3) }\end{array}$ & $\begin{array}{l}\text { 80th } \\
(4)\end{array}$ & $\begin{array}{l}\text { 95th } \\
\text { (5) }\end{array}$ & $\begin{array}{l}\text { 80th } \\
(6)\end{array}$ & $\begin{array}{l}\text { 80th } \\
(7)\end{array}$ & $\begin{array}{l}95 \text { th } \\
\text { (8) }\end{array}$ & $\begin{array}{l}\text { 80th } \\
\text { (9) }\end{array}$ & $\begin{array}{l}\text { 95th } \\
(10)\end{array}$ & $\begin{array}{l}\text { 80th } \\
\text { (11) }\end{array}$ & $\begin{array}{l}\text { 95th } \\
\text { (12) }\end{array}$ \\
\hline \multicolumn{13}{|l|}{ Life sciences } \\
\hline (1) Clinical medicine & 1.58 & 1.55 & 0.77 & 0.80 & 0.65 & 0.66 & 0.84 & 0.90 & 1.04 & 1.02 & 1.13 & 1.08 \\
\hline (2) Biology and biochemistry & 1.79 & 1.73 & 0.86 & 0.86 & 0.43 & 0.49 & 0.75 & 0.84 & 0.98 & 1.00 & 1.24 & 1.14 \\
\hline (3) Neuroscience and behav. science & 1.67 & 1.58 & 0.73 & 0.78 & 0.51 & 0.54 & 0.82 & 0.89 & 1.02 & 1.01 & 1.20 & 1.11 \\
\hline (4) Molecular biology and genetics & 1.53 & 1.48 & 0.77 & 0.81 & 0.58 & 0.60 & 0.82 & 0.90 & 0.99 & 1.00 & 1.24 & 1.13 \\
\hline (5) Psychiatry and psychology & 1.38 & 1.31 & 0.61 & 0.73 & 0.61 & 0.64 & 0.93 & 0.96 & 1.04 & 1.02 & 1.12 & 1.07 \\
\hline (6) Pharmacology and toxicology & 1.55 & 1.57 & 1.16 & 1.11 & 0.52 & 0.55 & 0.84 & 0.88 & 0.96 & 0.98 & 1.13 & 1.09 \\
\hline (7) Microbiology & 1.88 & 1.75 & 0.80 & 0.88 & 0.48 & 0.51 & 0.75 & 0.84 & 0.94 & 0.97 & 1.27 & 1.16 \\
\hline (8) Immunology & 1.35 & 1.34 & 0.66 & 0.74 & 0.98 & 0.88 & 0.83 & 0.90 & 1.04 & 1.03 & 1.16 & 1.09 \\
\hline \multicolumn{13}{|l|}{ Physical sciences } \\
\hline (9) Chemistry & 1.93 & 1.99 & 1.23 & 1.16 & 0.51 & 0.53 & 0.72 & 0.81 & 0.85 & 0.93 & 1.19 & 1.11 \\
\hline (10) Physics & 2.17 & 2.08 & 0.93 & 0.96 & 0.55 & 0.57 & 0.80 & 0.87 & 0.92 & 0.95 & 1.14 & 1.08 \\
\hline (11) Computer science & 1.61 & 1.61 & 0.51 & 0.53 & 0.94 & 0.92 & 0.89 & 0.92 & 1.01 & 1.01 & 1.09 & 1.06 \\
\hline (12) Mathematics & 2.18 & 1.97 & 0.70 & 0.81 & 0.50 & 0.54 & 0.89 & 0.93 & 0.93 & 0.96 & 1.13 & 1.08 \\
\hline (13) Space science & 1.37 & 1.39 & 0.99 & 0.98 & 0.63 & 0.63 & 0.77 & 0.87 & 1.02 & 1.01 & 1.21 & 1.12 \\
\hline \multicolumn{13}{|l|}{ Other natural sciences } \\
\hline (14) Engineering & 1.75 & 1.62 & 1.00 & 1.04 & 0.55 & 0.61 & 0.94 & 0.95 & 0.93 & 0.96 & 1.08 & 1.06 \\
\hline (15) Plant and animal science & 1.71 & 1.57 & 0.99 & 1.07 & 0.57 & 0.60 & 0.89 & 0.93 & 0.90 & 0.94 & 1.14 & 1.09 \\
\hline (16) Materials science & 2.51 & 2.27 & 0.89 & 0.94 & 0.57 & 0.62 & 0.84 & 0.89 & 0.92 & 0.96 & 1.09 & 1.06 \\
\hline (17) Geoscience & 1.55 & 1.55 & 0.99 & 0.98 & 0.62 & 0.63 & 0.79 & 0.87 & 0.90 & 0.96 & 1.23 & 1.13 \\
\hline (18) Environment and ecology & 1.64 & 1.52 & 0.90 & 0.94 & 0.53 & 0.60 & 0.92 & 0.94 & 0.93 & 0.96 & 1.13 & 1.08 \\
\hline (19) Agricultural sciences & 1.78 & 1.67 & 1.18 & 1.17 & 0.46 & 0.52 & 0.83 & 0.90 & 0.86 & 0.92 & 1.20 & 1.12 \\
\hline (20) Multidisciplinary & 2.58 & 2.52 & 1.05 & 1.12 & 0.33 & 0.33 & 0.69 & 0.79 & 0.91 & 0.93 & 1.16 & 1.11 \\
\hline \multicolumn{13}{|l|}{ Social sciences } \\
\hline (21) Social sciences, general & 1.39 & 1.33 & 0.67 & 0.74 & 0.42 & 0.49 & 0.95 & 0.97 & 1.00 & 1.01 & 1.11 & 1.07 \\
\hline (22) Economics and business & 1.52 & 1.50 & 0.61 & 0.62 & 0.43 & 0.46 & 0.89 & 0.92 & 1.06 & 1.04 & 1.16 & 1.10 \\
\hline
\end{tabular}

*In any field and any column, a cell value is greater than, equal to, or smaller than one when the geographical area's observed contribution (OC) to the world high- or low-impact level is greater than, equal to, or smaller than the area's publication share (or expected contribution, EC) in the extended citation distribution.

On the other hand, one of the properties of the FGT families of low-impact indicators is that when the CCL is raised, say from $z$ to $z^{\prime}$, for any $\beta$ the low-impact level of any citation distribution necessarily decreases; that is, $L_{\beta}(\boldsymbol{x} ; z)>L_{\beta}\left(\boldsymbol{x} ; z^{\prime}\right)$ for all $\boldsymbol{x}$ and $\beta$. However, for any particular area $k$, the relationship between the ratio $L_{2}\left(\boldsymbol{x}^{\boldsymbol{k}} ; z^{\prime}\right) / L_{2}\left(\boldsymbol{x} ; z^{\prime}\right)$ and $L_{2}\left(\boldsymbol{x}^{\boldsymbol{k}} ; z\right) / L_{2}(\boldsymbol{x} ; z)$ is again an empirical question.

Note that in the right-hand side of Table 5 (columns 7-12) the order in which the results are presented has changed: now the results for the 80th percentile come before than those for the 95th percentile. Very briefly, the U.S. OC/EC ratio increases, and hence the U.S. performance worsens, as the CCL increases in all 22 fields (compare columns 7 and 8 in Table 5). The same is the case for the EU in 15 fields; therefore, in the remaining seven disciplines the relative situation of the EU improves or remains constant (columns 9 and 10). However, since these improvements are really minimal, it turns out that the OC/EC ratio of the RW improves in all sciences (columns 11 and 12). Not surprisingly, such an improvement is greater when the contribution of the RW to the low-impact levels is large at the lower CCL, and when the worsening of the U.S. and the EU situations is greater (as in Biology and Biochemistry, Neuroscience and Behavioral Sciences, Molecular Biology and Genetics, Microbiology, Space Science, Agricultural Sciences, and Geosciences).

As we saw in Section 3.3, the change of the CCL from the 80th to the 95th percentile has large consequences for the volume of citations accounted for by low- and high-impact articles: for many sciences the percentage of total citations received by high-impact articles is reduced from 60-70\% to 27-35\% (columns 3 and 6 in Table 3). However, with some exceptions, it can be concluded that the impact of this change on the relative positions of the three geographical areas is relatively small.

\subsection{The effect of international co-authorship ${ }^{7}$}

As indicated in Section 1, this issue has two parts. Firstly, how do internationally co-authored articles fare versus domestic articles, namely, those written by people working in institutions belonging to just one of the three geographical areas?

\footnotetext{
7 The growth of international scientific collaboration has commanded a lot of attention (see inter alia, Frame \& Carpenter, 1979; Katz and Martin, 1997; Luukkonen, Persson, \& Sivertsen (1992); Van Raan, 1997, and the references contained in Luukkonen, Tijssen, Persson, \& Sivertsen, 1993). For the many reasons that motivate this complex phenomenon, and some reflections about the future, see in particular Beaver (2001) and the references therein.
} 
Table 6

The contribution of authorship types to high-impact worldwide levels in the original citation distribution for every scientific field ${ }^{\mathrm{a}}$ parameter $\beta=2$, and critical citation line $z=80$ th percentile of the original citation distribution.

\begin{tabular}{|c|c|c|c|c|c|c|c|}
\hline & $\begin{array}{l}\text { U.S. } \\
(1)\end{array}$ & $\begin{array}{l}\text { EU } \\
(2)\end{array}$ & $\begin{array}{l}\text { RW } \\
(3)\end{array}$ & $\begin{array}{l}\text { U.S + EU } \\
(4)\end{array}$ & $\begin{array}{l}\text { U.S. + RW } \\
\text { (5) }\end{array}$ & $\begin{array}{l}\text { EU + RW } \\
(6)\end{array}$ & $\begin{array}{l}\text { U.S. + EU + RW + RW } \\
\text { (7) }\end{array}$ \\
\hline \multicolumn{8}{|l|}{ Life sciences } \\
\hline (1) Clinical medicine & 1.56 & 0.55 & 0.32 & 3.01 & 1.74 & 1.30 & 13.71 \\
\hline (2) Biology and biochemistry & 1.94 & 0.75 & 0.28 & 1.50 & 1.12 & 0.92 & 3.65 \\
\hline (3) Neurosc. and behav. science & 1.65 & 0.58 & 0.27 & 2.28 & 1.63 & 0.85 & 4.10 \\
\hline (4) Mol. biology and genetics & 1.52 & 0.58 & 0.34 & 2.05 & 1.50 & 0.68 & 2.47 \\
\hline (5) Psychiatry and psychology & 1.31 & 0.58 & 0.37 & 2.34 & 1.88 & 0.94 & 3.21 \\
\hline (6) Pharma. and toxicology & 1.50 & 1.09 & 0.40 & 2.63 & 2.26 & 0.84 & 3.77 \\
\hline (7) Microbiology & 1.75 & 0.76 & 0.28 & 2.83 & 2.02 & 0.67 & 2.48 \\
\hline (8) Immunology & 1.40 & 0.56 & 0.74 & 1.58 & 1.33 & 1.01 & 1.99 \\
\hline \multicolumn{8}{|l|}{ Physical sciences } \\
\hline (9) Chemistry & 1.95 & 1.11 & 0.35 & 1.41 & 0.87 & 0.48 & 37.94 \\
\hline (10) Physics & 2.09 & 0.66 & 0.24 & 2.70 & 2.05 & 0.62 & 10.57 \\
\hline (11) Computer science & 0.49 & 0.30 & 0.09 & 6.70 & 11.39 & 0.13 & 0.34 \\
\hline (12) Mathematics & 2.02 & 0.60 & 0.34 & 3.33 & 2.02 & 0.59 & 2.85 \\
\hline (13) Space science & 1.09 & 0.49 & 0.09 & 2.57 & 1.42 & 0.38 & 4.04 \\
\hline \multicolumn{8}{|l|}{ Other natural sciences } \\
\hline (14) Engineering & 1.65 & 0.88 & 0.51 & 3.20 & 1.14 & 1.02 & 6.44 \\
\hline (15) Plant and animal science & 1.56 & 1.02 & 0.46 & 2.98 & 1.54 & 0.91 & 3.94 \\
\hline (16) Materials science & 2.41 & 0.94 & 0.54 & 1.94 & 2.11 & 0.66 & 6.02 \\
\hline (17) Geosciences & 1.49 & 0.62 & 0.22 & 2.28 & 1.22 & 0.61 & 13.98 \\
\hline (18) Environment and ecology & 1.55 & 0.79 & 0.39 & 2.85 & 1.28 & 1.01 & 6.04 \\
\hline (19) Agricultural sciences & 1.58 & 1.13 & 0.41 & 5.20 & 1.65 & 0.95 & 4.76 \\
\hline (20) Multidisciplinary & 2.65 & 0.73 & 0.14 & 5.44 & 3.34 & 1.11 & 5.03 \\
\hline \multicolumn{8}{|l|}{ Social sciences } \\
\hline (21) Social sciences, general & 1.34 & 0.61 & 0.31 & 2.79 & 1.40 & 1.05 & 5.08 \\
\hline (22) Economics and business & 1.56 & 0.35 & 0.16 & 2.53 & 1.26 & 0.71 & 2.30 \\
\hline
\end{tabular}

a In any field and any column, a cell value is greater than, equal to, or smaller than one when the authorship type's observed contribution (OC) to the world high-impact level is greater than, equal to, or smaller than the type's publication share (or expected contribution EC) in the extended citation distribution.

Secondly, what role do internationally co-authored articles play in each of the areas? For the first question we should work with the original distributions in all fields. For reasons of space we will exclusively discuss the high-impact aspects. As already indicated, the information about the FGT indices for the seven subgroups in the partition by authorship type, as well as for the world as a whole, are in columns 1-8 in Table A in the Appendix. However, the ratios $H_{2}\left(\boldsymbol{x}^{\boldsymbol{j}} ; ; z\right) / H_{2}(\boldsymbol{x} ; ; z)$ for $j=1, \ldots, 7$ and for a CCL fixed at the 80th percentile, are in Table 6 (results on low-impact levels are available on request).

As has been repeatedly observed in the literature, international co-authorship as a whole is vastly successful. ${ }^{8}$ However, in our data set there are some differences worth emphasizing. Firstly, except for Computer Science, the OC according to $\mathrm{H}_{2}$ is always greater than the EC for articles written in the three areas (column 7 in Table 6). In particular, the ratio OC/EC is in the $(1,5)$ interval in 12 fields, in the $(5,7)$ interval in five fields, and greater than seven in four remarkable cases: 10.6 in Physics, 13.7 in Clinical Medicine, 14 in Geosciences, and 37.9 in Chemistry (!). This is a clear indication of the presence in this group of those articles receiving the highest number of citations that, from a statistical point of view, often constitute extreme observations. Secondly, the cooperation between the U.S. and the EU is almost as successful, with the OC/EC ratio in the interval $[1.4,3.2]$ in nine fields, in the interval $(5,6)$ in two cases, and equal to 6.7 in Computer Science (column 4). Thirdly, the cooperation between the U.S. and the RW yields also rather favorable results with the ratio OC/EC in the $(1,2)$ interval in 16 cases, in the $(2,3)$ interval in four cases, and equal to 11.4 in Computer Science (column 5). Fourthly, things are considerably different in the cooperation between the EU and the RW (column 6). The OC/EC ratio for this type is only clearly above one in three fields (Clinical Medicine, Multidisciplinary, and Social Sciences, General). ${ }^{9}$ Finally, the correlation coefficient between the publication effort (columns 4-7 in Table 1) and the corresponding $\mathrm{H}_{2}\left(\boldsymbol{x}^{j}, z\right)$ levels for co-authorship types (columns 4-7 in Table $A$ in the Appendix) are far from being significant. For instance, the Spearman rank correlation coefficient are $-0.04,-0,02,-0.003$, and -0.31 , respectively. Thus, a greater publication effort in a field by a co-authorship type does not guarantee a high-impact level in that field.

\footnotetext{
8 In the words of Glänzel and Schubert (2001), "the increase of citation impact by international collaboration became almost a commonplace notion". See inter alia, Narin et al. (1991), REIST-2 (1997), Glänzel et al. (1999) and Glänzel (2000, 2001).

9 That international co-authorship not always pays for all partners has been also found in previous studies. See, inter alia, Glänzel and Schubert (2001) who referred to this phenomenon as cool links, as well as Glänzel et al. (1999), and Glänzel (2001).
} 
Table 7

The contribution of authorship type subgroups to high-impact geographical area levels in the expanded citation distribution for every scientific field ${ }^{a}$ parameter $\beta=2$, and critical citation line $z=80$ th percentile of the original citation distr bution (1) the relative contribution of articles written in the U.S. (EU or RW) alone to the high-impact level of the U.S. (EU or RW); (2) the relative contribution of articles co-authored in the U.S. and the EU (EU and U.S., or U.S. and RW) to the high-impact level of the U.S. (EU or RW); (3) the relative contribution of articles co-authored in the U.S. and the RW (EU and RW, or RW and EU)) to the high-impact level of the U.S. (EU or RW); (4) the relative contribution of articles co-authored in the U.S., the EU, and the RW to the high-impact level of the U.S. (EU or RW).

\begin{tabular}{|c|c|c|c|c|c|c|c|c|c|c|c|c|}
\hline & \multicolumn{4}{|c|}{ United States } & \multicolumn{4}{|c|}{ European Union } & \multicolumn{4}{|c|}{ Rest of the world } \\
\hline \multicolumn{13}{|l|}{ Life sciences } \\
\hline (2) Biology and biochemistry & 1.06 & 0.82 & 0.61 & 2.00 & 0.82 & 1.64 & 1.01 & 4.01 & 0.54 & 2.19 & 1.79 & 7.13 \\
\hline (3) Neuroscience and behavioral science & 0.94 & 1.29 & 0.93 & 2.33 & 0.67 & 2.62 & 0.98 & 4.73 & 0.44 & 2.70 & 1.41 & 6.79 \\
\hline (4) Molecular biology and genetics & 0.94 & 1.27 & 0.93 & 1.52 & 0.66 & 2.31 & 0.76 & 2.77 & 0.51 & 2.28 & 1.03 & 3.74 \\
\hline (5) Psychiatry/psychology & 0.93 & 1.67 & 1.34 & 2.29 & 0.75 & 3.00 & 1.20 & 4.11 & 0.54 & 2.75 & 1.37 & 4.68 \\
\hline (8) Immunology & 0.98 & 1.10 & 0.93 & 1.39 & 0.71 & 2.00 & 1.27 & 2.51 & 0.79 & 1.43 & 1.08 & 2.13 \\
\hline \multicolumn{13}{|l|}{ Physical sciences } \\
\hline (9) Chemistry & 0.79 & 0.57 & 0.35 & 15.32 & 0.76 & 0.97 & 0.33 & 26.11 & 0.53 & 1.32 & 0.73 & 57.35 \\
\hline (10) Physics & 0.75 & 0.98 & 0.74 & 3.82 & 0.51 & 2.10 & 0.48 & 8.23 & 0.32 & 2.72 & 0.82 & 14.01 \\
\hline (11) Computer science & 0.19 & 2.68 & 4.56 & 0.13 & 0.37 & 8.20 & 0.16 & 0.41 & 0.06 & 7.96 & 0.09 & 0.23 \\
\hline (12) Mathematics & 0.91 & 1.51 & 0.91 & 1.29 & 0.66 & 3.65 & 0.65 & 3.13 & 0.55 & 3.30 & 0.97 & 4.66 \\
\hline (16) Material science & 1.01 & 0.81 & 0.89 & 2.52 & 0.95 & 1.96 & 0.67 & 6.08 & 0.82 & 3.23 & 1.01 & 9.19 \\
\hline (17) Geosciences & 0.71 & 1.09 & 0.58 & 6.65 & 0.46 & 1.71 & 0.46 & 10.46 & 0.26 & 1.43 & 0.72 & 16.45 \\
\hline (18) Environment and ecology & 0.93 & 1.71 & 0.77 & 3.61 & 0.76 & 2.74 & 0.97 & 5.81 & 0.58 & 1.93 & 1.51 & 9.07 \\
\hline (19) Agricultural sciences & 0.88 & 2.88 & 0.91 & 2.63 & 0.89 & 4.11 & 0.75 & 3.76 & 0.72 & 2.92 & 1.67 & 8.41 \\
\hline (20) Multidisciplinary & 0.89 & 1.82 & 1.12 & 1.68 & 0.54 & 4.08 & 0.83 & 3.77 & 0.35 & 8.43 & 2.79 & 12.69 \\
\hline \multicolumn{13}{|l|}{ Social sciences } \\
\hline (21) Social sciences, general & 0.97 & 2.02 & 1.01 & 3.68 & 0.80 & 3.62 & 1.36 & 6.60 & 0.61 & 2.73 & 2.04 & 9.89 \\
\hline (22) Economics and business & 0.97 & 1.57 & 0.78 & 1.43 & 0.52 & 3.79 & 1.07 & 3.45 & 0.33 & 2.57 & 1.45 & 4.69 \\
\hline
\end{tabular}

${ }^{a}$ In any field and any column, a cell value is greater than, equal to, or smaller than one when the authorship type's observed contribution (OC) to the world high-impact level is greater than, equal to, or smaller than the type's publication share (or expected contribution EC) in the extended citation distribution.

By way of contrast, the domestic performance of the EU and the RW is much less successful: the OC/EC ratio is above one only in four fields (Plant and Animal Science, Pharmacology and Toxicology, Chemistry, and Agricultural Sciences) in the EU and for no field at all in the RW (columns 2 and 3 in Table 6). The situation of domestic articles written in the U.S. is considerably different: the ratio OC/EC is below one only in Computer Science, in the interval $(1,2)$ in 17 fields and equal to or greater than two in four remarkable cases (Mathematics, Physics, Materials Science, and Multidisciplinary). Nevertheless, it is worth emphasizing that this ratio for the U.S. domestic articles is always smaller than at least one of the three co-authorship types involving the U.S. itself.

For the second part of this issue, it is necessary to get back to the extended citation distributions. For a given area, say the U.S., the ratios $\mathrm{H}_{2}\left(\boldsymbol{x}^{j} ; z\right) / \mathrm{H}_{2}(\boldsymbol{x} ; z)$ for $j=$ domestic U.S. articles, U.S. + EU, U.S. + RW, and U.S. + EU + RW are presented in Table 7. The first thing to note is that there are two fields in which international co-authorship is essential: Computer Science, where the cooperation between the U.S. and the EU, and the U.S. and the RW is totally dominant, and Space Science, where the cooperation between the U.S. and the EU, and between the three areas is equally important. In the second place, when the U.S. domestic articles compete with those internationally co-authored within the expanded count, the ratio OC/EC for the U.S. alone is equal to or above one in only two fields (biology and biochemistry, and materials science). Moreover, in both cases this ratio is below the one for the subgroup in which the three areas cooperate. This indicates that, even for the U.S., international co-authorship tends to be decisive. ${ }^{10}$

This conclusion cannot but be reinforced for the EU. The ratio OC/EC for the EU domestic articles is always below one. This means that in each of the six fields in which the EU as a whole contributes to the overall high-impact levels above what could be expected from its publication share in Table 4A (Agricultural Sciences, Chemistry, Multidisciplinary, Pharmacology and Toxicology, Plant and Animal Science, and Engineering), the explanation lies in international co-authorship. As a matter of fact, international co-authorship is so important for the EU that, without it, the OC/EC ratio of the domes-

\footnotetext{
10 That international co-authorship increases citation attractivity in comparison to the already high domestic standard in countries such as Denmark and New Zealand has also been documented in Glänzel (2001).
} 
tic EU articles is far below one in fields as important as Geosciences (0.46), Physics (0.51), Economics and Business (0.52), Clinical Medicine (0.56), Mathematics (0.66), and Molecular Biology and Genetics (0.66). Finally, the RW domestic articles perform dramatically worse in every single field than those written in collaboration with the U.S., or with both the U.S. and the EU.

\section{Conclusions}

This paper has presented the first empirical applications of a novel method to evaluate the impact of research units in a homogeneous scientific field. Given a CCL fixed at a sufficiently high percentage of the world citation distribution, the procedure calls for the computation of a pair of high- and low-impact indicators to describe key features of a research unit's citation distribution. A companion paper (Albarrán et al., 2010c) provides a strong justification for the use of the FGT families of high- and low-impact indicators. These indicators have been applied to the citation distributions of 22 fields, consisting of articles published in 1998-2002 with a five-year citation window. The remainder of this concluding section summarizes the main results of this investigation, and briefly comments on a number of possible extensions.

\subsection{Main results}

Among the main results, a distinction is made between those of a methodological and a substantive type. From a methodological point of view three points should be mentioned:

1. The additive decomposability property of the two FGT families has provided a convenient framework, never used before in the bibliometric literature, in the study of three related partitions: the partition of the original world-wide citation distribution in each field into seven authorship types (Table 6), the partition of the extended distributions in three large geographical areas by authorship type (Table 7), and the partition of the extended distribution for the world as a whole into the three geographical areas, namely, the U.S., the EU, and the RW (Tables 4A and 4B and Figs. 1 and 2). In each case, the observed contribution of any subgroup to the overall high- or low-impact level is compared to the subgroup's expected contribution that coincides with its publication share in the field in question.

2. The approach advocated in this paper emphasizes the distinction between the incidence, the intensity, and the inequality of the high- and the low-impact aspects of any citation distribution. The results in Section 4.1 indicate that to use parameter values $\beta=0,1$, or 2 to identify the members of the FGT families that successfully capture these dimensions make a considerable empirical difference.

3. Quite unexpectedly, in Section 4.3 it was found that, as far as the high-impact is concerned, raising the CCL from the 80th to the 95th percentile of the world citation distribution does not dramatically alter the relative situation of geographical areas and/or scientific fields - a fact that would have to be checked among smaller research units and scientific sub-fields, as well as for even higher CCLs.

Among substantive results, the following four will be emphasized:

4. The U.S. occupies a truly enviable position in the two directions investigated in this paper: its observed contribution to the world high-impact level in the extended citation distribution is greater than what is expected from its publication share in all fields; and its relative contribution to the world low-impact level is also smaller than expected in all fields. Part of this success is indeed due to the international cooperation with the other two areas. In the partition of the U.S. stock of articles into domestic and internationally co-authored publications, the contribution to the U.S. domestic articles to the U.S. high-impact level is always smaller than that of some of the types of international co-authorship. Nevertheless, in the partition of the world articles into the three domestic subgroups and the four types of international co-authorship, the contribution of the U.S. domestic articles to the overall high-impact level is greater than what is expected from its publication share in 21 of the 22 fields - showing again the outstanding performance of the research done in U.S. institutions.

5. By comparison, the situation of the EU is worrisome. Its contribution to the world high-impact level is strictly above its publication share in the extended distribution in only six fields (Agricultural Sciences, Chemistry, Multidisciplinary, Pharmacology and Toxicology, Plant and Animal Science, and Engineering), while its contribution to the world low-impact level is equal to or above its expected value in as many as eight fields. Furthermore, in all fields with a relatively good performance the EU success is very dependent on the international cooperation with the U.S. alone or with the U.S. and the RW simultaneously.

6. Perhaps not surprisingly, the situation of the very heterogeneous area RW is not good at all. Its best result indicates that its observed contribution to the world high-impact level in Computer Science and Immunology is $12 \%$ and $8 \%$ below what can be expected from its publication share in that field's extended citation distribution. But even this modest performance is due to the international cooperation with the U.S., or the U.S. and the EU simultaneously. Finally, it should be noted that the articles co-authored by the EU and the RW make a positive contribution only in six of the 22 fields (Clinical Medicine, Immunology, Engineering, Environment and Ecology, Multidisciplinary, and Social Sciences, General). 
7. The absolute number of articles authored by the RW is considerably larger than that of the EU or the U.S. in 13 fields. In turn, more articles are written in the EU than in the U.S. in 14 fields. Together with points 4-6, this indicates that an area's large publication share within a field is no guarantee at all of a good high- or low-impact performance. At the same time, publication efforts across fields within a geographical area are also unrelated to good high- or low-impact performances.

\subsection{Possible extensions}

This first attempt at applying a certain methodology to a large dataset admits several extensions, among which the following six should be mentioned. Firstly, it is important to know how this approach fares versus the alternatives. In Albarrán et al. (2010c), this paper's results are compared with those that can be obtained for each geographical area using the alternative with better properties, namely, what we call the Leiden triad of indicators consisting of the mean citation rate, and the area's percentage contribution to the set of uncited papers and to the top $5 \%$ of highly cited papers in a given field.

Secondly, the analysis of international co-authorship has been very cursory. The unconditional comparisons between domestic and international articles have not even controlled for the number of authors as in Persson, Glänzel, and Danell (2004). Furthermore, a proper treatment of this issue would have to explicitly model the self-selection aspect of this phenomenon pointed out in Narin, Stevens, and Whitlow (1991) and Glänzel and Schubert (2001). On the other hand, a study of links strength, countries clustering, or the distinction between intra- and international cooperation in the EU would require the extension of this example to the many countries case.

Thirdly, just as the analysis can be extended to more countries or, more generally, to a larger set of research units, it can obviously be extended to a greater number of scientific sub-fields.

Fourthly, this framework can be profitably used for the analysis of inter-temporal trends. Recall that, for any partition, overall high- or low-impact levels can be expressed as the weighted average of each subgroup's high- or low-impact levels, where the weights are the subgroups' publication shares. Therefore, inter-temporal comparisons of overall levels can be accounted for by changes in publication share and by changes in subgroups' index values. A simple application of this decomposition can be found in Ortuño and Ruiz-Castillo (2010a).

Fifthly, one could think of many contexts in which having two statistics to describe a citation distribution's shape is very appropriate. However, it must be admitted that often the greatest interest is focused on the upper tail of citation distributions in a search for what can described as research excellence. In this respect note that, by raising the CCL, high-impact indicators increasingly become indicators of research excellence. But, as pointed out in Section 4.4, FGT and similar high-impact indices are very sensitive to extreme observations, namely, to the presence of one or a few articles with a phenomenal number of citations. In a sense, this is as it should be, because these articles do exist and the indices should reflect them. But at the same time one is also interested in completing our high-impact results with those obtained using robust indicators of research excellence, such as the $h$-index, first suggested by Hirsh (2005) for the evaluation of individual scientists but very soon used also for the evaluation of all sorts of research units. Thus, as indicated in Albarrán et al. (2010c), the $h$-index and the procedures discussed in this paper have different properties, serve different purposes, and therefore constitute essentially complementary approaches to the same evaluation problem.

Finally, the approach presented in this paper can be questioned on two grounds. In the first place, ultimately 'any choice of a single measure. . is apt to be arbitrary' (Foster, 1984, p. 242), and so are the conclusions based on this measure. In the second place, any method to fix a CCL also includes a significant degree of arbitrariness. Since a high- or a low-impact measure may produce contradictory conclusions at two different yet equally reasonable CCLs, the use of a single CCL is also arbitrary, and so are the conclusions based upon this procedure. Borrowing results from the poverty literature in economics, Ortuño and Ruiz-Castillo (2010b) address these two issues for the measurement of both the high- and low-impact aspects of citation distributions.

\section{Acknowledgements}

The authors acknowledge financial support by Santander Universities Global Division of Banco Santander. Albarrán and Ruiz-Castillo also acknowledge financial help from the Spanish MEC, the first through grants SEJ2007-63098 and SEJ2006-05710, and the second through grant SEJ2007-67436. Finally, this paper is part of the SCIFI-GLOW Collaborative Project supported by the European Commission's Seventh Research Framework Programme, Contract number SSH7-CT-2008-217436. Comments and suggestions by Juan A. Crespo and one referee are gratefully acknowledged.

\section{Appendix.}

See Table A. 
Table A

High- and low-impact FGT indicators for parameter values $\beta=0,1,2$, by co-authorship type and scientific field. Critical citation line $=80$ th percentile of the original distribution.

\begin{tabular}{|c|c|c|c|c|c|c|c|c|c|}
\hline & $\begin{array}{l}\text { U.S. } \\
\text { (1) }\end{array}$ & $\begin{array}{l}\mathrm{EU} \\
(2)\end{array}$ & $\begin{array}{l}\text { RW } \\
\text { (3) }\end{array}$ & $\begin{array}{l}\text { U.S + EU } \\
(4)\end{array}$ & $\begin{array}{l}\text { U.S. + RW } \\
\text { (5) }\end{array}$ & $\begin{array}{l}\mathrm{EU}+\mathrm{RW} \\
(6)\end{array}$ & $\begin{array}{l}\text { U.S. + EU + RW } \\
\text { (7) }\end{array}$ & $\begin{array}{l}\text { Total original } \\
\text { (8) }\end{array}$ & $\begin{array}{l}\text { Total extended } \\
\text { (9) }\end{array}$ \\
\hline \multicolumn{10}{|l|}{ (1) Clinical medicine } \\
\hline$\beta=0$ & 0.25 & 0.17 & 0.12 & 0.40 & 0.32 & 0.26 & 0.50 & 0.19 & 0.21 \\
\hline$\beta=2$ & 3.63 & 1.29 & 0.76 & 7.00 & 4.04 & 3.02 & 31.90 & 2.33 & 2.86 \\
\hline$\beta=0$ & 0.75 & 0.83 & 0.88 & 0.60 & 0.68 & 0.74 & 0.50 & 0.81 & 0.79 \\
\hline Low-impact $\beta=1$ & 0.49 & 0.59 & 0.65 & 0.34 & 0.42 & 0.46 & 0.27 & 0.56 & 0.54 \\
\hline$\beta=0$ & 0.28 & 0.17 & 0.10 & 0.32 & 0.26 & 0.16 & 0.35 & 0.19 & 0.20 \\
\hline High-impact $\beta=1$ & 0.31 & 0.15 & 0.08 & 0.36 & 0.28 & 0.16 & 0.45 & 0.19 & 0.20 \\
\hline$\beta=2$ & 1.56 & 0.60 & 0.22 & 1.21 & 0.90 & 0.74 & 2.94 & 0.81 & 0.85 \\
\hline$\beta=0$ & 0.72 & 0.83 & 0.90 & 0.68 & 0.74 & 0.84 & 0.65 & 0.81 & 0.80 \\
\hline Low-impact $\beta=1$ & 0.42 & 0.55 & 0.67 & 0.37 & 0.45 & 0.55 & 0.36 & 0.54 & 0.53 \\
\hline$\beta=2$ & 0.31 & 0.42 & 0.55 & 0.26 & 0.33 & 0.42 & 0.25 & 0.42 & 0.41 \\
\hline$\beta=0$ & 0.74 & 0.83 & 0.90 & 0.68 & 0.74 & 0.80 & 0.62 & 0.81 & 0.80 \\
\hline Low-impact $\beta=1$ & 0.44 & 0.54 & 0.63 & 0.37 & 0.44 & 0.49 & 0.33 & 0.52 & 0.51 \\
\hline$\beta=2$ & 0.32 & 0.41 & 0.50 & 0.25 & 0.32 & 0.36 & 0.23 & 0.39 & 0.38 \\
\hline \multicolumn{10}{|c|}{ (4) Molecular biology and genetics } \\
\hline$\beta=0$ & 0.26 & 0.16 & 0.09 & 0.34 & 0.25 & 0.19 & 0.40 & 0.20 & 0.21 \\
\hline High-impact $\beta=1$ & 0.36 & 0.18 & 0.10 & 0.48 & 0.36 & 0.21 & 0.58 & 0.25 & 0.28 \\
\hline$\beta=2$ & 1.47 & 0.56 & 0.33 & 1.98 & 1.45 & 0.65 & 2.38 & 0.96 & 1.06 \\
\hline$\beta=0$ & 0.74 & 0.84 & 0.91 & 0.66 & 0.75 & 0.81 & 0.60 & 0.80 & 0.79 \\
\hline Low-impact $\beta=1$ & 0.46 & 0.57 & 0.70 & 0.39 & 0.48 & 0.55 & 0.34 & 0.54 & 0.53 \\
\hline$\beta=2$ & 0.35 & 0.44 & 0.58 & 0.28 & 0.37 & 0.42 & 0.24 & 0.43 & 0.41 \\
\hline \multicolumn{10}{|c|}{ (5) Psychiatry and psychology } \\
\hline$\beta=0$ & 0.21 & 0.17 & 0.12 & 0.36 & 0.27 & 0.24 & 0.43 & 0.19 & 0.44 \\
\hline$\beta=2$ & 0.80 & 0.58 & 0.21 & 1.41 & 1.21 & 0.45 & 2.01 & 0.53 & 0.58 \\
\hline$\beta=0$ & 0.74 & 0.81 & 0.89 & 0.66 & 0.76 & 0.81 & 0.61 & 0.82 & 0.81 \\
\hline Low-impact $\beta=1$ & 0.46 & 0.53 & 0.61 & 0.37 & 0.47 & 0.51 & 0.33 & 0.54 & 0.53 \\
\hline$\beta=2$ & 0.35 & 0.41 & 0.48 & 0.27 & 0.36 & 0.39 & 0.24 & 0.42 & 0.41 \\
\hline \multicolumn{10}{|l|}{ (7) Microbiology } \\
\hline$\beta=0$ & 0.29 & 0.19 & 0.09 & 0.35 & 0.25 & 0.20 & 0.34 & 0.20 & 0.21 \\
\hline High-impact $\beta=1$ & 0.29 & 0.16 & 0.06 & 0.40 & 0.26 & 0.16 & 0.39 & 0.18 & 0.19 \\
\hline$\beta=2$ & 0.69 & 0.30 & 0.11 & 1.12 & 0.80 & 0.27 & 0.98 & 0.40 & 0.44 \\
\hline$\beta=0$ & 0.71 & 0.81 & 0.91 & 0.65 & 0.75 & 0.80 & 0.66 & 0.80 & 0.79 \\
\hline Low-impact $\beta=1$ & 0.40 & 0.50 & 0.65 & 0.34 & 0.45 & 0.48 & 0.34 & 0.51 & 0.50 \\
\hline$\beta=2$ & 0.28 & 0.37 & 0.53 & 0.23 & 0.33 & 0.35 & 0.23 & 0.38 & 0.37 \\
\hline \multicolumn{10}{|l|}{ (8) Immunology } \\
\hline$\beta=0$ & 0.25 & 0.16 & 0.11 & 0.32 & 0.24 & 0.19 & 0.30 & 0.20 & 0.21 \\
\hline High-impact $\beta=1$ & 0.28 & 0.15 & 0.11 & 0.36 & 0.28 & 0.22 & 0.38 & 0.20 & 0.22 \\
\hline$\beta=2$ & 0.93 & 0.37 & 0.49 & 1.05 & 0.89 & 0.67 & 1.32 & 0.67 & 0.71 \\
\hline$\beta=0$ & 0.75 & 0.84 & 0.89 & 0.68 & 0.76 & 0.81 & 0.70 & 0.80 & 0.79 \\
\hline Low-impact $\beta=1$ & 0.44 & 0.55 & 0.62 & 0.37 & 0.45 & 0.50 & 0.38 & 0.51 & 0.50 \\
\hline$\beta=2$ & 0.31 & 0.42 & 0.49 & 0.26 & 0.32 & 0.37 & 0.26 & 0.38 & 0.37 \\
\hline \multicolumn{10}{|l|}{ (9) Chemistry } \\
\hline$\beta=0$ & 0.33 & 0.22 & 0.12 & 0.37 & 0.27 & 0.20 & 0.32 & 0.20 & 0.20 \\
\hline High-impact $\beta=1$ & 0.47 & 0.23 & 0.12 & 0.47 & 0.31 & 0.20 & 0.53 & 0.22 & 0.23 \\
\hline$\beta=2$ & 2.02 & 1.15 & 0.36 & 1.46 & 0.90 & 0.50 & 39.23 & 1.03 & 1.29 \\
\hline$\beta=0$ & 0.67 & 0.78 & 0.88 & 0.63 & 0.73 & 0.80 & 0.68 & 0.80 & 0.80 \\
\hline Low-impact $\beta=1$ & 0.41 & 0.49 & 0.65 & 0.35 & 0.46 & 0.51 & 0.38 & 0.55 & 0.54 \\
\hline$\beta=2$ & 0.31 & 0.37 & 0.54 & 0.25 & 0.35 & 0.40 & 0.28 & 0.44 & 0.43 \\
\hline
\end{tabular}




\begin{tabular}{|c|c|c|c|c|c|c|c|c|c|}
\hline & $\begin{array}{l}\text { U.S. } \\
(1)\end{array}$ & $\begin{array}{l}\text { EU } \\
(2)\end{array}$ & $\begin{array}{l}\text { RW } \\
(3)\end{array}$ & $\begin{array}{l}\text { U.S + EU } \\
(4)\end{array}$ & $\begin{array}{l}\text { U.S. + RW } \\
\text { (5) }\end{array}$ & $\begin{array}{l}\text { EU + RW } \\
(6)\end{array}$ & $\begin{array}{l}\text { U.S. + EU + RW } \\
(7)\end{array}$ & $\begin{array}{l}\text { Total original } \\
(8)\end{array}$ & $\begin{array}{l}\text { Total extended } \\
\text { (9) }\end{array}$ \\
\hline \multicolumn{10}{|l|}{ (10) Physics } \\
\hline$\beta=0$ & 0.27 & 0.20 & 0.10 & 0.33 & 0.26 & 0.20 & 0.38 & 0.18 & 0.19 \\
\hline High-impact $\beta=1$ & 0.48 & 0.28 & 0.12 & 0.59 & 0.47 & 0.28 & 0.85 & 0.27 & 0.30 \\
\hline$\beta=2$ & 4.00 & 1.26 & 0.46 & 5.17 & 3.93 & 1.18 & 20.23 & 1.91 & 2.55 \\
\hline$\beta=0$ & 0.73 & 0.80 & 0.90 & 0.67 & 0.74 & 0.80 & 0.62 & 0.82 & 0.81 \\
\hline Low-impact $\beta=1$ & 0.49 & 0.56 & 0.69 & 0.41 & 0.49 & 0.55 & 0.37 & 0.59 & 0.57 \\
\hline$\beta=2$ & 0.39 & 0.45 & 0.58 & 0.32 & 0.39 & 0.44 & 0.28 & 0.49 & 0.47 \\
\hline \multicolumn{10}{|l|}{ (11) Computer science } \\
\hline$\beta=0$ & 0.23 & 0.16 & 0.12 & 0.31 & 0.26 & 0.18 & 0.36 & 0.18 & 0.19 \\
\hline High-impact $\beta=1$ & 0.53 & 0.28 & 0.17 & 0.91 & 0.72 & 0.30 & 0.70 & 0.36 & 0.39 \\
\hline$\beta=2$ & 5.53 & 3.40 & 1.00 & 76.15 & 129.50 & 1.47 & 3.81 & 11.37 & 17.67 \\
\hline$\beta=0$ & 0.77 & 0.84 & 0.88 & 0.69 & 0.74 & 0.82 & 0.64 & 0.82 & 0.81 \\
\hline Low-impact $\beta=1$ & 0.56 & 0.63 & 0.69 & 0.47 & 0.52 & 0.58 & 0.43 & 0.62 & 0.61 \\
\hline$\beta=2$ & 0.49 & 0.56 & 0.62 & 0.39 & 0.44 & 0.49 & 0.36 & 0.54 & 0.53 \\
\hline \multicolumn{10}{|l|}{ (12) Mathematics } \\
\hline$\beta=0$ & 0.19 & 0.17 & 0.10 & 0.29 & 0.22 & 0.19 & 0.30 & 0.16 & 0.17 \\
\hline High-impact $\beta=1$ & 0.30 & 0.20 & 0.10 & 0.47 & 0.31 & 0.22 & 0.47 & 0.21 & 0.22 \\
\hline$\beta=2$ & 1.83 & 0.54 & 0.31 & 3.02 & 1.83 & 0.54 & 2.58 & 0.91 & 1.02 \\
\hline$\beta=0$ & 0.81 & 0.83 & 0.90 & 0.71 & 0.78 & 0.81 & 0.70 & 0.84 & 0.83 \\
\hline Low-impact $\beta=1$ & 0.57 & 0.59 & 0.70 & 0.43 & 0.51 & 0.54 & 0.40 & 0.61 & 0.59 \\
\hline$\beta=2$ & 0.48 & 0.49 & 0.62 & 0.34 & 0.42 & 0.45 & 0.31 & 0.52 & 0.50 \\
\hline \multicolumn{10}{|l|}{ (13) Space science } \\
\hline$\beta=0$ & 0.27 & 0.15 & 0.06 & 0.35 & 0.26 & 0.15 & 0.37 & 0.20 & 0.22 \\
\hline High-impact $\beta=1$ & 0.32 & 0.15 & 0.05 & 0.48 & 0.34 & 0.14 & 0.61 & 0.24 & 0.28 \\
\hline$\beta=2$ & 1.16 & 0.52 & 0.09 & 2.75 & 1.51 & 0.41 & 4.32 & 1.07 & 1.42 \\
\hline$\beta=0$ & 0.73 & 0.85 & 0.94 & 0.65 & 0.74 & 0.85 & 0.63 & 0.80 & 0.78 \\
\hline Low-impact $\beta=1$ & 0.46 & 0.63 & 0.75 & 0.38 & 0.46 & 0.58 & 0.36 & 0.56 & 0.53 \\
\hline$\beta=2$ & 0.36 & 0.53 & 0.65 & 0.28 & 0.35 & 0.46 & 0.27 & 0.46 & 0.42 \\
\hline \multicolumn{10}{|l|}{ (14) Engineering } \\
\hline$\beta=0$ & 0.20 & 0.18 & 0.12 & 0.30 & 0.22 & 0.23 & 0.35 & 0.17 & 0.18 \\
\hline High-impact $\beta=1$ & 0.31 & 0.23 & 0.14 & 0.50 & 0.29 & 0.28 & 0.70 & 0.22 & 0.24 \\
\hline$\beta=2$ & 1.42 & 0.76 & 0.44 & 2.76 & 0.99 & 0.88 & 5.55 & 0.86 & 0.94 \\
\hline$\beta=0$ & 0.80 & 0.82 & 0.88 & 0.70 & 0.78 & 0.77 & 0.65 & 0.83 & 0.82 \\
\hline Low-impact $\beta=1$ & 0.58 & 0.58 & 0.66 & 0.44 & 0.52 & 0.52 & 0.40 & 0.60 & 0.59 \\
\hline$\beta=2$ & 0.49 & 0.49 & 0.57 & 0.35 & 0.43 & 0.42 & 0.31 & 0.51 & 0.50 \\
\hline \multicolumn{10}{|c|}{ (15) Plant and animal science } \\
\hline$\beta=0$ & 0.20 & 0.20 & 0.11 & 0.35 & 0.24 & 0.22 & 0.37 & 0.17 & 0.18 \\
\hline High-impact $\beta=1$ & 0.25 & 0.23 & 0.10 & 0.52 & 0.30 & 0.22 & 0.59 & 0.19 & 0.21 \\
\hline$\beta=2$ & 1.01 & 0.67 & 0.30 & 1.94 & 1.00 & 0.59 & 2.56 & 0.65 & 0.70 \\
\hline$\beta=0$ & 0.80 & 0.80 & 0.89 & 0.65 & 0.76 & 0.78 & 0.63 & 0.83 & 0.82 \\
\hline Low-impact $\beta=1$ & 0.53 & 0.53 & 0.65 & 0.36 & 0.47 & 0.49 & 0.34 & 0.57 & 0.55 \\
\hline$\beta=2$ & 0.42 & 0.42 & 0.55 & 0.27 & 0.36 & 0.38 & 0.24 & 0.46 & 0.45 \\
\hline \multicolumn{10}{|l|}{ (16) Material science } \\
\hline$\beta=0$ & 0.27 & 0.22 & 0.15 & 0.34 & 0.26 & 0.22 & 0.35 & 0.20 & 0.20 \\
\hline High-impact $\beta=1$ & 0.52 & 0.30 & 0.19 & 0.58 & 0.47 & 0.28 & 0.65 & 0.29 & 0.30 \\
\hline$\beta=2$ & 3.40 & 1.33 & 0.76 & 2.74 & 2.98 & 0.93 & 8.49 & 1.41 & 1.49 \\
\hline$\beta=0$ & 0.73 & 0.78 & 0.85 & 0.66 & 0.74 & 0.78 & 0.65 & 0.80 & 0.80 \\
\hline Low-impact $\beta=1$ & 0.49 & 0.54 & 0.63 & 0.41 & 0.49 & 0.52 & 0.42 & 0.57 & 0.56 \\
\hline$\beta=2$ & 0.40 & 0.45 & 0.54 & 0.32 & 0.40 & 0.42 & 0.33 & 0.48 & 0.47 \\
\hline \multicolumn{10}{|l|}{ (17) Geosciences } \\
\hline$\beta=0$ & 0.27 & 0.20 & 0.09 & 0.35 & 0.28 & 0.21 & 0.41 & 0.20 & 0.21 \\
\hline High-impact $\beta=1$ & 0.31 & 0.18 & 0.08 & 0.40 & 0.31 & 0.20 & 0.72 & 0.20 & 0.23 \\
\hline$\beta=2$ & 1.01 & 0.42 & 0.15 & 1.55 & 0.83 & 0.41 & 9.50 & 0.68 & 0.92 \\
\hline$\beta=0$ & 0.73 & 0.80 & 0.91 & 0.65 & 0.72 & 0.79 & 0.59 & 0.80 & 0.79 \\
\hline Low-impact $\beta=1$ & 0.45 & 0.52 & 0.69 & 0.36 & 0.44 & 0.50 & 0.32 & 0.54 & 0.52 \\
\hline$\beta=2$ & 0.35 & 0.40 & 0.59 & 0.26 & 0.33 & 0.38 & 0.23 & 0.44 & 0.42 \\
\hline \multicolumn{10}{|c|}{ (18) Environment and ecology } \\
\hline$\beta=0$ & 0.21 & 0.18 & 0.11 & 0.34 & 0.25 & 0.20 & 0.39 & 0.18 & 0.19 \\
\hline High-impact $\beta=1$ & 0.22 & 0.16 & 0.09 & 0.39 & 0.25 & 0.19 & 0.60 & 0.17 & 0.18 \\
\hline$\beta=2$ & 0.75 & 0.38 & 0.19 & 1.37 & 0.62 & 0.48 & 2.89 & 0.48 & 0.53 \\
\hline$\beta=0$ & 0.79 & 0.82 & 0.89 & 0.66 & 0.75 & 0.80 & 0.61 & 0.82 & 0.81 \\
\hline Low-impact $\beta=1$ & 0.52 & 0.52 & 0.63 & 0.36 & 0.46 & 0.50 & 0.33 & 0.55 & 0.53 \\
\hline \multirow[t]{2}{*}{$\beta=2$} & 0.40 & 0.40 & 0.52 & 0.25 & 0.34 & 0.37 & 0.23 & 0.43 & 0.42 \\
\hline & & & & & & & & & 22 \\
\hline
\end{tabular}




\begin{tabular}{|c|c|c|c|c|c|c|c|c|c|}
\hline & $\begin{array}{l}\text { U.S. } \\
(1)\end{array}$ & $\begin{array}{l}\text { EU } \\
(2)\end{array}$ & $\begin{array}{l}\text { RW } \\
\text { (3) }\end{array}$ & $\begin{array}{l}\text { U.S + EU } \\
(4)\end{array}$ & $\begin{array}{l}\text { U.S. + RW } \\
\text { (5) }\end{array}$ & $\begin{array}{l}\mathrm{EU}+\mathrm{RW} \\
(6)\end{array}$ & $\begin{array}{l}\text { U.S. + EU + RW } \\
\text { (7) }\end{array}$ & $\begin{array}{l}\text { Total original } \\
(8)\end{array}$ & $\begin{array}{l}\text { Total extended } \\
\text { (9) }\end{array}$ \\
\hline \multicolumn{10}{|c|}{ (19) Agricultural sciences } \\
\hline$\beta=0$ & 0.25 & 0.24 & 0.12 & 0.34 & 0.24 & 0.24 & 0.36 & 0.19 & 0.20 \\
\hline High-impact $\beta=1$ & 0.32 & 0.26 & 0.11 & 0.59 & 0.30 & 0.25 & 0.63 & 0.22 & 0.23 \\
\hline$\beta=2$ & 1.04 & 0.74 & 0.27 & 3.41 & 1.08 & 0.62 & 3.12 & 0.66 & 0.71 \\
\hline$\beta=0$ & 0.75 & 0.76 & 0.88 & 0.66 & 0.76 & 0.76 & 0.64 & 0.81 & 0.80 \\
\hline Low-impact $\beta=1$ & 0.49 & 0.49 & 0.67 & 0.36 & 0.46 & 0.48 & 0.31 & 0.56 & 0.55 \\
\hline$\beta=2$ & 0.39 & 0.39 & 0.57 & 0.26 & 0.34 & 0.37 & 0.23 & 0.46 & 0.45 \\
\hline \multicolumn{10}{|l|}{ (20) Multidisciplinary } \\
\hline$\beta=0$ & 0.34 & 0.22 & 0.07 & 0.42 & 0.29 & 0.20 & 0.56 & 0.17 & 0.18 \\
\hline High-impact $\beta=1$ & 0.99 & 0.48 & 0.10 & 1.59 & 0.87 & 0.42 & 2.04 & 0.41 & 0.46 \\
\hline$\beta=2$ & 11.63 & 3.18 & 0.60 & 23.88 & 14.67 & 4.85 & 22.09 & 4.39 & 5.21 \\
\hline$\beta=0$ & 0.66 & 0.78 & 0.93 & 0.58 & 0.71 & 0.80 & 0.44 & 0.83 & 0.82 \\
\hline Low-impact $\beta=1$ & 0.47 & 0.62 & 0.75 & 0.38 & 0.48 & 0.57 & 0.29 & 0.65 & 0.63 \\
\hline$\beta=2$ & 0.40 & 0.55 & 0.68 & 0.31 & 0.40 & 0.48 & 0.24 & 0.58 & 0.56 \\
\hline \multicolumn{10}{|c|}{ (21) Social sciences, general } \\
\hline$\beta=0$ & 0.20 & 0.16 & 0.11 & 0.30 & 0.24 & 0.23 & 0.39 & 0.18 & 0.18 \\
\hline High-impact $\beta=1$ & 0.29 & 0.18 & 0.11 & 0.51 & 0.32 & 0.29 & 0.77 & 0.23 & 0.24 \\
\hline$\beta=2$ & 1.14 & 0.52 & 0.27 & 2.38 & 1.20 & 0.89 & 4.33 & 0.85 & 0.89 \\
\hline$\beta=0$ & 0.80 & 0.84 & 0.89 & 0.70 & 0.76 & 0.77 & 0.61 & 0.82 & 0.82 \\
\hline Low-impact $\beta=1$ & 0.56 & 0.60 & 0.67 & 0.43 & 0.49 & 0.49 & 0.35 & 0.59 & 0.58 \\
\hline$\beta=2$ & 0.47 & 0.51 & 0.58 & 0.34 & 0.39 & 0.39 & 0.27 & 0.50 & 0.49 \\
\hline \multicolumn{10}{|c|}{ (22) Economics and Business } \\
\hline$\beta=0$ & 0.22 & 0.11 & 0.07 & 0.32 & 0.22 & 0.14 & 0.29 & 0.17 & 0.18 \\
\hline High-impact $\beta=1$ & 0.35 & 0.11 & 0.06 & 0.53 & 0.31 & 0.16 & 0.51 & 0.24 & 0.25 \\
\hline$\beta=2$ & 1.41 & 0.31 & 0.14 & 2.28 & 1.14 & 0.64 & 2.08 & 0.90 & 0.97 \\
\hline$\beta=0$ & 0.78 & 0.89 & 0.93 & 0.68 & 0.78 & 0.86 & 0.71 & 0.83 & 0.82 \\
\hline Low-impact $\beta=1$ & 0.54 & 0.66 & 0.73 & 0.42 & 0.52 & 0.61 & 0.41 & 0.60 & 0.59 \\
\hline$\beta=2$ & 0.45 & 0.56 & 0.64 & 0.33 & 0.41 & 0.51 & 0.30 & 0.51 & 0.49 \\
\hline
\end{tabular}

As indicated in Section 4, the last two columns contain two indicators at the overall, aggregate level whose construction deserve some explanation. Consider, for example, the first row in field (1), Clinical Medicine, corresponding to the FGT index $H_{0}$. The first overall index is simply the sum of the seven subgroups' indicators weighted by the subgroups publication shares taken from Table 1 . The result listed in column 8 is 0.19 for $H_{0}$. This is in spite of the fact that the CCL is the 80th percentile of the original citation distribution, which means that the high-and low-impact indicators when $\beta=0, H_{0}$ and $L_{0}$, should be equal to 0.20 and 0.80 . The reason is that the CCL is typically rather low (see Table 3 ), and there is a large number of articles receiving exactly that low number of citations, which in the Clinical Medicine case is 13 . Thus, it is natural to classify all these articles as low-impact ones, raising their percentage - i.e. raising $L_{0}$ - above 0.80 and, correspondingly, lowering the percentage of high-impact articles - i.e. $H_{0}-$ below 0.20 .

The second index aggregates the indicators for the three geographical areas in the extended count. Thus, it is the sum of the areas' indicators weighted by the areas' publication shares taken from the right-hand side of Table 2. In turn, each area's indicator is the weighted sum of the corresponding subgroups' indicators. For example, the U.S. index in a given field is constructed by aggregating the indicators of the four subgroups formed by articles (i) written exclusively in the U.S., co-authored by (ii) U.S. and EU researchers, (iii) U.S. and RW researchers, and (iv) researchers from the three areas. The weights used in the aggregation are the publication shares of these four subgroups in the U.S. total. For instance, in Clinical Medicine in the U.S. case the number of articles in these subgroups are 229,$151 ; 20,442 ; 25,050$, and 5,133 (see Table 1 ) with a total of 279,776 . Therefore, the publication shares $81.9 ; 7.3 ; 9.0$, and 1.8 are used to weight the four $H_{0}$ indicators $0.25,0.40,0.31$, and 0.50 taken from the corresponding columns in the row identified by $\beta=00$ in this Appendix. The resulting weighted sum for the U.S. is 0.27 . Computing the corresponding magnitudes for the EU and the RW, the final weighted sum for Clinical Medicine as a whole is 0.21 , the figure that appears in column 9 in the row $\beta=0$.

\section{References}

Albarrán, P., \& Ruiz-Castillo, J. (2009). “References Made and Citations Received By Scientific Articles”, Working Paper 09-81, Economics Series 45, Universidad Carlos III, in press in Journal of the American Society for Information Science and Technology.

Albarrán, P., Crespo, J., Ortuño, I., \& Ruiz-Castillo, J. (2010a). A Comparison of the Scientific Performance of the U.S. and Europe at the Turn of the 21st Century. Scientometrics, $85,329-344$.

Albarrán, P., Crespo, J., Ortuño, I., \& Ruiz-Castillo, J. (2010b). The Skewness of Science in 219 Sub-fields and a Number of Aggregates, Working Paper 10-16, Universidad Carlos III.

Albarrán, P., Ortuño, I., \& Ruiz-Castillo, J. (2010c). Average-based versus high- and low-impact indicators for the evaluation of citation distributions with, Working Paper 10-10. Universidad Carlos III.

Albarrán, P., Ortuño, I., \& Ruiz-Castillo, J. (2011). The measurement of low- and high-impact in citation distributions: technical results. Journal of Informetrics, $5,48-63$.

Anderson, J., Collins, P., Irvine, J., Isard, P., Martin, B., Narin, F., \& Stevens, K. (1988). On-line approaches to measuring national scientific output: A cautionary tale. Science and Public Policy, 15, 153-161.

deB Beaver, D. (2001). Reflections on scientific collaboration (and its study): Past, present, and future. Scientometrics, $52,365-377$.

Egghe, L., Rousseau, R., \& Rousseau, S. (2007). TOP-Curves. Journal of the American Society for Information Science and Technology, $58,777-785$.

Foster, J. E. (1984). On economic poverty: A survey of aggregate measures, in advances in econometrics, vol. 3. JAI Press Inc., pp. 215-251

Foster, J. E., Greeer, J., \& Thorbecke, E. (1984). A class of decomposable poverty measures. Econometrica, 52, 761-766.

Frame, J., \& Carpenter, M. (1979). International research collaboration. Social Studies of Science, 9, 481-497.

Glänzel, W. (2000). Science in Scandinavia: A bibliometric approach. Scientometrics, 48, 121-150.

Glänzel, W. (2001). National characteristics in international scientific co-authorship relations. Scientometrics, 51, 69-115.

Glänzel, W. (2007). Characteristic scores and scales. A bibliometric analysis of subject characteristics based on long-term citation observation. Journal of Informetrics, 1, 92-102. 
Glänzel, W., \& Schubert, A. (2001). Double effort = double impact? A critical view at international co-authorship in chemistry. Scientometrics, $51,69-115$. Glänzel, W., Schubert, A., \& Czerwon, H.-J. (1999). A bibliometric analysis of international scientific cooperation of the European Union (1985-1995). Scientometrics, 45, 185-202.

Hirsch, J. (2005). An index to quantify an individual's scientific research output. Proceedings of the National Academy of Sciences of the United Status of America, $16569-16572,102$.

Katz, S., \& Martin, B. R. (1997). What is research collab? Research Policy, 26, 1-18.

King, D. (2004). The scientific impact of nations. Nature, 430, 311-316.

Luukkonen, T., Persson, O., \& Sivertsen, G. (1992). Understanding patterns of international scientific collaboration. Science, Technology, and Human Values, $17,101-126$.

Luukkonen, T., Tijssen, R. J. W., Persson, O., \& Sivertsen, G. (1993). The measurement of international scientific collaboration. Scientometrics, 28, 15-36.

May, R. (1997). The scientific wealth of nations. Science, 275, 793-796.

Narin, F., Stevens, K., \& Whitlow, E. (1991). Scientific co-operation in Europe and the citation of multinationally authored papers. Scientometrics, 21 , $313-323$.

Ortuño, I., \& Ruiz-Castillo, J. (2010a). Trends in high- and low-impact levels in citation distributions, 1998-2004, mimeo.

Ortuño, I., \& Ruiz-Castillo, J. (2010b). Dominance results for the measurement of low- and high-impact in citation distributions, mimeo.

Persson, O., Glänzel, W., \& Danell, R. (2004). Inflationary bibliometric values: The role of scientific collaboration and the need for relative indicators in evaluation studies. Scientometrics, 60, 421-432.

REIST-2 (1997). The European Report on Science and Technology Indicators 1997, EUR 17639, European Commission, Brussels.

Rousseau, S., Verbeke, T., \& Rousseau, R. (2009). Evaluating environmental and resource economics journals: A TOP-Curve approach. Review of Environmental Economics and Policy, 3, 270-287.

Schubert, A., Glänzel, W., \& Braun, T. (1987). A new methodology for ranking scientific institutions. Scientometrics, 12, $267-292$.

Van Raan, A. F. J. (1997). Science as an international enterprise. Science and Public Policy, 24, 290-300. 